\title{
Entropy Stable Method for the Euler Equations Revisited: Central Differencing via Entropy Splitting and SBP
}

\author{
Björn Sjögreen and H.C.Yee
}

April 14, 2019

\begin{abstract}
The two decades old high order central differencing via entropy splitting and summation-by-parts (SBP) difference boundary closure of Olsson \& Oliger (1994), Gerritsen \& Olsson (1996), and Yee et al. (2000) is revisited. The entropy splitting is a form of skew-symmetric splitting of the nonlinear Euler flux derivatives. Central differencing applied to the entropy splitting form of the Euler flux derivatives together with SBP difference operators will, hereafter, be referred to as entropy split schemes. This study is prompted by the recent growing interest in numerical methods for which a discrete entropy conservation law holds, a discrete global entropy conservation can be proved and/or the numerical method possesses a stable entropy in the framework of SBP difference operators and $L_{2}$-energy norm estimate. The objective of this paper is to recast the entropy split scheme as the recent definition of an entropy stable method for central differencing with SBP operators for both periodic and non-periodic boundary conditions for nonlinear Euler equations. Standard high order spatial central differencing as well as high order central spatial DRP (dispersion relation preserving) spatial differencing is part of the entropy stable methodology framework. Long time integration of $2 \mathrm{D}$ and 3D test cases is included to show the comparison of this efficient entropy stable method with the Tadmor-type of entropy conservative methods. Studies also include the comparison among the three skew-symmetric splittings on their nonlinear stability and accuracy performance without added numerical dissipations for smooth flows. These are, namely, entropy splitting, Ducros et al. splitting and the Kennedy \& Grubber splitting.
\end{abstract}

Björn Sjögreen

Multid Analyses AB, Address of Institute, e-mail: bjorn.sjogreen@multid.se

H.C.Yee

NASA Ames Research Center, Address of Institute e-mail: Helen.C.Yee@nas.nasa.gov 


\section{Introduction and objectives}

Recently there has been growing interest in numerical methods for which a discrete entropy conservation law holds, a discrete global entropy conservation can be proved, and/or the numerical method possesses a stable entropy in the framework of summation-by-parts (SBP) and $L_{2}$-energy norm estimate. These growing research topics recently attracted, in particular, the discontinuous Galerkin method development community. See [6, 2] and references cited therein for some related development. Their focus is on the Tadmor-type of entropy conservative and or entropy stable methods [29, 30, 31]. See Ranocha for an overview [17]. For the Tadmor-type entropy stable methods development, often diffusive numerical dissipation terms (e.g., entropy viscosity) are needed to prove stability.

One of the reasons for interest in stable entropy numerical methods is the advantage of improving nonlinear stability and accuracy of simulations for nonlinear fluid flow problems. The improved nonlinear stability in turn would help minimize the used of added numerical dissipation and thus improve numerical accuracy. As discussed in [17], this is still a topic that needs further research. Our main interest here is focused on improving nonlinear stability of long time integration of shock-free turbulence and turbulence with shocks for the gas dynamics and the MHD equations. Nonlinearly stable methods that are suitable for rapidly developing or for shorter time integration of fluid flows might still be suffering from nonlinear instability for longer time integration of, e.g., shock-free turbulence and turbulence with shocks computations. In the direct numerical simulations (DNS) and large eddy simulations (LES) research circle, reducing aliasing errors based mainly on the linearized approach has been developed to improve nonlinear stability of long time integration of DNS and LES computations.

In this article the two decades old high order central differencing via entropy splitting and SBP difference closure of Olsson \& Oliger (1994), Gerritsen \& Olsson (1996), and Yee et al. (2000) [15, 7, 37] is revisited. This is an expanded version of a short proceedings paper reported in [26]. The entropy splitting is a form of skew-symmetric splitting of the nonlinear Euler flux derivatives. Central differencing applied to the entropy splitting form of the Euler flux derivatives together with SBP difference operators will, hereafter, be referred to as entropy split schemes. Here we recast the entropy split scheme as the recent definition of an entropy stable method for central differencing with SBP operators for both periodic and non-periodic boundary conditions for nonlinear Euler equations. An entropy split scheme satisfies the $L_{2}$-energy norm estimate readily without an added numerical dissipation term for smooth flows. Standard high order spatial central differencing as well as high order central spatial DRP (dispersion relation preserving) spatial differencing is part of the entropy stable methodology. For flows containing discontinuities the Yee et al. nonlinear filter approach $[36,37,38,39,25,24]$ is employed at isolated computed locations, after each full time step of the 
entropy split method to suppress spurious oscillations while maintaining accuracy on the remaining flow field. Since the nonlinear filter step is executed as a Euler time discretization at isolated location after the completion of full time step of the entropy stable central scheme, entropy conservation/stability is valid almost everywhere.

The first objective of this paper is a revisit to show in the current popular definition of entropy stability based on the $L^{2}$ norm that entropy splitting using the physical entropy for central schemes with SBP operators are entropy stable. Standard high order spatial central differencing as well as high order central spatial DRP spatial differencing are included in the numerical studies. The second objective is to illustrate the efficiency and performance of the split schemes with the more CPU intensive Tadmor-type entropy conserving methods. 2D and 3D long time integration of smooth flows, shock-free turbulence and turbulence with shocklets are included. Comparisons among the three skew-symmetric splittings (entropy splitting [37], Ducros et al. splitting [3] and the Kennedy \& Grubber splitting [12]) on their nonlinear stability and accuracy performance without added numerical dissipations for smooth flows is included. Entropy split methods are defined for a family of entropies that depend on a parameter, $\beta$. The influence of $\beta$ on accuracy and stability of the resulting numerical method was briefly studied in [37, 27]. Here, the third objective is to show the influence of $\beta$ for two 3D DNS test cases. Furthermore, since the entropy split methods are not in conservative form, another goal of the current study is to investigate how these methods perform on the same two 3D turbulent test cases with and without discontinuities.

\section{Preliminaries}

Consider the system of conservation laws

$$
\mathbf{q}_{t}+\mathbf{f}_{x}=\mathbf{0},
$$

with flux function $\mathbf{f}(\mathbf{q})$. A convex function $E(\mathbf{q})$ is an entropy for (1) if the additional conservation law

$$
E_{t}+F_{x}=0
$$

where $F$ is the entropy flux, can be derived from (1) under smoothness assumptions. It is well-known that the existence of an entropy is equivalent with existence of a symmetrizing change of variables given by the entropy variables $\mathbf{v}=E_{\mathbf{q}}$; see [8] and references therein.

Now consider the conservation law (1) as the nonlinear Euler equations for a perfect gas, thermally perfect gas or a linear combination of perfect gases. The entropy split methods for the Euler equations by Olsson \& Oliger 
(1994), Gerritsen \& Olsson (1996), and Yee et al. (2000) $[15,7,37]$ that conserve the global entropy were developed in the 90's but have received less attention in recent years. As indicated in Olsson \& Oliger (1994), Gerritsen \& Olsson (1996), and Yee et al. (2000, for central spatial differencing, entropy split schemes are simple to define. All that is needed is to replace the spatial derivatives by summation-by-parts (SBP) difference operators in the entropy split form of the equations. The numerical boundary closure follows directly from the SBP operator. No additional numerical boundary procedure is required. In contrast, Tadmor-type entropy conserving schemes [30], and more recently in [35], do not naturally come with a numerical boundary closure. A generalized SBP operator has to be developed [18]. These boundary operators have been developed for Tadmor-type entropy conservative schemes; see [16]. They are more complicated to define and implement. The next section will briefly summarize the Olsson \& Oliger (1994), Gerritsen \& Olsson (1996), and Yee et al. (2000) work first before showing their entropy split schemes are entropy stable.

\subsection{Remarks}

Remark I: It is noted that the Hughes et al. formulation [9] using the Harten's idea [8] but solving the flow equations in nonconservative form in terms of the entropy variables is completely different from the entropy split schemes. The entropy split scheme solve the entropy splitting form of the Euler flux derivatives consisting of a one parameter family of conservative and a non-conservative portions in terms of the entropy variables. If the parameter satisfies the energy estimate, entropy stability is immediate.

Remark II: The entropy split scheme has been generalized from perfect gas to thermally perfect gas and gas flows consist of linear combination of perfect gases $[34,37]$. In addition, these high order schemes has been formulated in time varying deforming curvilinear grids with free-stream preservation [34, $21]$.

\subsection{Some Terminology}

In order to clarify some of the terminology we include some definitions that will be used in the following section. A method for solving the equations of gas dynamics is globally entropy conservative if the computed solution satisfies

$$
\Delta x \frac{d}{d t} \sum_{j=1}^{N} E_{j}(t)=0
$$


with periodic boundary conditions, or with boundary conditions

$$
\Delta x \frac{d}{d t} \sum_{j=1}^{N} \omega_{j} E_{j}(t)+F_{N}-F_{1}=0,
$$

where $F_{j}$ is the entropy flux at point $x_{j}$, and $\omega_{j}$ are quadrature weights. $F_{1}$ and $F_{N}$ are the two end points value of the entropy flux.

The term "entropy consistency" is sometimes used for "entropy conservation". A method for solving the gas dynamics equations is entropy dissipative, or "entropy stable", if the computed solution satisfies (3) or (4) with inequality,

$$
\Delta x \frac{d}{d t} \sum_{j=1}^{N} E_{j}(t) \leq 0,
$$

or

$$
\Delta x \frac{d}{d t} \sum_{j=1}^{N} \omega_{j} E_{j}(t)+F_{N}-F_{1} \leq 0 .
$$

A method for solving the gas dynamics equations is locally entropy conservative if there exists a numerical entropy flux function $H_{j+1 / 2}$ such that the computed solution satisfies a relation

$$
\frac{d}{d t} E_{j}(t)+\frac{1}{\Delta x}\left(H_{j+1 / 2}-H_{j-1 / 2}\right)=0, \quad j=1, \ldots, N .
$$

\section{Entropy of Gas dynamics Equations}

We consider the 3D equations of inviscid compressible gas dynamics

$$
\mathbf{q}_{t}+\mathbf{f}_{x}+\mathbf{g}_{x}+\mathbf{h}_{x}=\mathbf{0}
$$

with conserved variables

$$
\mathbf{q}=(\rho \rho u \rho v \rho w e)^{T}
$$

and fluxes in an arbitrary direction $\mathbf{k}=\left(\begin{array}{lll}k_{1} & k_{2} & k_{3}\end{array}\right)$ with $|\mathbf{k}|^{2}=1$, and

$$
\hat{\mathbf{f}}=k_{1} \mathbf{f}+k_{2} \mathbf{g}+k_{3} \mathbf{h}=\left(\rho \hat{u} \rho u \hat{u}+k_{1} p \rho v \hat{u}+k_{2} p \rho w \hat{u}+k_{3} p \hat{u}(e+p)\right)^{T},
$$

where $\hat{u}=k_{1} u+k_{2} v+k_{3} w$. The total energy is related to the pressure $p$ by the ideal gas law,

$$
e=\frac{p}{\gamma-1}+\frac{1}{2} \rho|\mathbf{u}|^{2},
$$

where $\gamma>1$ is a given constant, and $|\mathbf{u}|^{2}=u^{2}+v^{2}+w^{2}$. 
An entropy is a convex function, $E(\mathbf{q})$, of the conserved variables that allows an additional conservation law,

$$
E_{t}+F_{x}+G_{y}+H_{z}=0
$$

when the solution is smooth. The entropy fluxes in the $x$-, $y$-, and $z$-directions are denoted by $F, G$, and $H$, respectively. The entropy variables are defined by $\mathbf{v}=\nabla_{\mathbf{q}} E$ (the notation $E_{\mathbf{q}}$ for the gradient will sometimes be used). The convexity of $E$ ensures that these are well-defined. The Entropy conservation law (8) follows if the relation

$$
\mathbf{v}^{T} \frac{\partial \mathbf{f}}{\partial \mathbf{q}}=\nabla_{\mathbf{q}} F
$$

for the $x$-direction fluxes, and similarly for the $y$ - and $z$-directions, holds. Moreover, the entropy variables symmetrize the equations; $\partial \mathbf{f} / \partial \mathbf{v}$ is a symmetric matrix.

A. Harten [8] considered the class of entropies

$$
E=-\frac{\gamma+\alpha}{\gamma-1} \rho\left(p \rho^{-\gamma}\right)^{\frac{1}{\alpha+\gamma}},
$$

where $\alpha$ is a parameter. To ensure that $E$ is convex, i.e., that the matrix $E_{\mathbf{q}, \mathbf{q}}$ is positive definite, $\alpha$ is required to satisfy $\alpha>0$ or $\alpha<-\gamma$. The full range for $\alpha$ was given in [37], while [8] only considered $\alpha>0$, and [7] used only the special case $\alpha=1-2 \gamma$ from $\alpha<-\gamma$.

The corresponding entropy flux in the direction $\mathbf{k}=\left(\begin{array}{lll}k_{1} & k_{2} & k_{3}\end{array}\right)^{T}$ is

$$
F=\hat{u} E .
$$

The entropy variables $\mathbf{v}=E_{\mathbf{q}}$ are straightforwardly found to be

$$
\mathbf{v}=\frac{\rho}{p} s^{\frac{1}{\alpha+\gamma}}\left(-\frac{\alpha}{\gamma-1} \frac{p}{\rho}-\frac{1}{2}|\mathbf{u}|^{2} u v w-1\right)^{T},
$$

where $s$ denotes $p \rho^{-\gamma}$. The entropy split discretization relies on the homogeneity property given in the following lemma.

Lemma 1. The conserved variables are homogeneous functions of the entropy variables (10),

$$
\mathbf{q}(\theta \mathbf{v})=\theta^{\beta} \mathbf{q}(\mathbf{v}),
$$

where $\beta=(\alpha+\gamma) /(1-\gamma)$. From (11) it follows that

$$
\begin{aligned}
\mathbf{q}_{\mathbf{v}} \mathbf{v} & =\beta \mathbf{q} \\
\hat{\mathbf{f}}_{\mathbf{v}} \mathbf{v} & =\beta \hat{\mathbf{f}} .
\end{aligned}
$$

Proof. To prove (11), first note that 


$$
u=-v_{2} / v_{5} \quad v=-v_{3} / v_{5} \quad w=-v_{4} / v_{5},
$$

and, hence, that the velocities $(u, v, w)$ are invariant under the scaling $\theta \mathbf{v}$. Rewriting the first entropy variable as

$$
v_{1}=v_{5}\left(\frac{\alpha}{\gamma-1} \frac{p}{\rho}+\frac{1}{2}|\mathbf{u}|^{2}\right)
$$

shows that $p / \rho$ is invariant under the scaling $\theta \mathbf{v}$ as well. This invariance together with the definition of $v_{5}$ now give that

$$
s^{\frac{1}{\alpha+\gamma}}(\theta \mathbf{v})=\theta s^{\frac{1}{\alpha+\gamma}}(\mathbf{v}) .
$$

It holds that

$$
s^{\frac{1}{\alpha+\gamma}}=\left(p \rho^{-\gamma}\right)^{\frac{1}{\alpha+\gamma}}=\left(\frac{p}{\rho}\right)^{\frac{1}{\alpha+\gamma}}\left(\rho^{1-\gamma}\right)^{\frac{1}{\alpha+\gamma}} .
$$

Because $p / \rho$ is invariant, (14) gives

$$
\rho^{\frac{1-\gamma}{\alpha+\gamma}}(\theta \mathbf{v})=\theta \rho^{\frac{1-\gamma}{\alpha+\gamma}}(\mathbf{v})
$$

which means that

$$
\rho(\theta \mathbf{v})=\theta^{\frac{\alpha+\gamma}{1-\gamma}} \rho(\mathbf{v})
$$

By defining $\beta=\frac{\alpha+\gamma}{1-\gamma}$, the conclusion $\rho(\theta \mathbf{v})=\theta^{\beta} \rho(\mathbf{v})$ follows. Because $u, v, w$, and $p / \rho$ are invariant, the conserved variables scale in the same way as $\rho$, thereby proving (11). The flux homogeneity follows from the homogeneity of the Euler fluxes in conserved variables, $\hat{\mathbf{f}}(\theta \mathbf{q})=\theta \hat{\mathbf{f}}$, and from (11) by

$$
\hat{\mathbf{f}}(\theta \mathbf{v})=\hat{\mathbf{f}}(\mathbf{q}(\theta \mathbf{v}))=\hat{\mathbf{f}}\left(\theta^{\beta} \mathbf{q}(\mathbf{v})\right)=\theta^{\beta} \hat{\mathbf{f}}(\mathbf{q}(\mathbf{v}))=\theta^{\beta} \hat{\mathbf{f}}(\mathbf{v})
$$

The relations (12) and (13) are obtained by differentiating (11) and (15) with respect to $\theta$ and setting $\theta=1$.

Remark: The range of $\alpha$, where $E_{\mathbf{q}, \mathbf{q}}$ is positive definite, translates to $\beta$ satisfying

$$
\beta<-\frac{\gamma}{\gamma-1} \text { or } \beta>0
$$

The entropy splitting described in the next section weights the nonconservative portion of the flux derivative by $\frac{1}{1+\beta}$. This means that the range $\beta>0$ corresponds to a weight that is less than 1 , whereas negative $\beta$ leads, unphysically, to a weight that is greater than 1 . 


\section{Entropy splitting of the Euler flux derivative}

This section reviews some of the results from Gerritsen \& Olsson and Yee et al. [7,37]. Consider the one dimensional gas dynamics equations on $a<x<b$,

$$
\mathbf{q}_{t}+\mathbf{f}_{x}=\mathbf{0} \text {. }
$$

The flux derivative is written as a weighted sum of a conservative part, $\mathbf{f}_{x}$, and a non-conservative part, $\mathbf{f}_{\mathbf{v}} \mathbf{v}_{x}$, as

$$
\mathbf{f}_{x}=\frac{\beta}{\beta+1} \mathbf{f}_{x}+\frac{1}{\beta+1} \mathbf{f}_{\mathbf{v}} \mathbf{v}_{x}
$$

Replacing $\mathbf{f}_{x}$ in (16) by this split flux derivative gives

$$
\mathbf{q}_{t}+\frac{\beta}{\beta+1} \mathbf{f}_{x}+\frac{1}{\beta+1} \mathbf{f}_{\mathbf{v}} \mathbf{v}_{x}=\mathbf{0} .
$$

Equation (17) is the entropy split form of (16). Introduce the scalar product

$$
(\mathbf{u}, \mathbf{v})=\int_{a}^{b} \mathbf{u}^{T} \mathbf{v} d x
$$

The time derivative of the global entropy over $a<x<b$ becomes

$$
\begin{gathered}
\frac{d}{d t} \int_{a}^{b} E(\mathbf{q}) d x=\left(\mathbf{v}, \mathbf{q}_{t}\right)=-\frac{\beta}{\beta+1}\left(\mathbf{v}, \mathbf{f}_{x}\right)-\frac{1}{\beta+1}\left(\mathbf{v}, \mathbf{f}_{\mathbf{v}} \mathbf{v}_{x}\right)= \\
\frac{\beta}{\beta+1}\left(\mathbf{v}_{x}, \mathbf{f}\right)-\frac{\beta}{\beta+1}\left(\mathbf{v}(b, t)^{T} \mathbf{f}(b, t)-\mathbf{v}(a, t)^{T} \mathbf{f}(a, t)\right)-\frac{1}{\beta+1}\left(\mathbf{f}_{\mathbf{v}} \mathbf{v}, \mathbf{v}_{x}\right)
\end{gathered}
$$

where we used integration-by-parts on the $\beta /(\beta+1)$-term, and the symmetry of $\mathbf{f}_{\mathbf{v}}$ on the $1 /(\beta+1)$-term. The flux homogeneity (13) eliminates all terms except the boundary contributions,

$$
\frac{d}{d t} \int_{a}^{b} E(\mathbf{q}) d x=-\frac{\beta}{\beta+1}\left(\mathbf{v}(b, t)^{T} \mathbf{f}(b, t)-\mathbf{v}(a, t)^{T} \mathbf{f}(a, t)\right)
$$

This is an entropy balance law, because

$$
\frac{\beta}{\beta+1} \mathbf{v}^{T} \mathbf{f}=F
$$

so that the right hand side of (19) is just the difference of entropy fluxes across the boundaries $x=a$ and $x=b$.

Equation (20) can be proved by direct evaluation using (7) and (10). This leads to 
Title Suppressed Due to Excessive Length

$$
\mathbf{v}^{T} \mathbf{f}=\frac{\rho}{p} s^{\frac{1}{\alpha+\gamma}}\left(-\frac{\alpha+1}{\gamma-1} \hat{u} p\right)=-\frac{\alpha+1}{\gamma-1} \rho \hat{u} s^{\frac{1}{\alpha+\gamma}} .
$$

From the definition of $\beta$ it holds that

$$
\frac{\beta}{\beta+1}=\frac{\alpha+\gamma}{\alpha+1}
$$

Hence,

$$
\frac{\beta}{\beta+1} \mathbf{v}^{T} \mathbf{f}=-\frac{\alpha+\gamma}{\alpha+1} \cdot \frac{\alpha+1}{\gamma-1} \rho \hat{u} s^{\frac{1}{\alpha+\gamma}}=-\frac{\alpha+\gamma}{\gamma-1} \rho \hat{u} s^{\frac{1}{\alpha+\gamma}}=\hat{u} E=F .
$$

The global entropy conservation (19) can be rewritten as an $L^{2}$-like estimate. The following result was given in [7].

Lemma 2. The entropy time derivative can be rewritten as

$$
\frac{d}{d t} E(\mathbf{q})=\frac{1}{\beta+1} \frac{d}{d t}\left(\mathbf{v}^{T}\left(E_{\mathbf{q}, \mathbf{q}}\right)^{-1} \mathbf{v}\right)
$$

by using the homogeneity (11).

Proof. By definition $\mathbf{v}=E_{\mathbf{q}}$ and $\mathbf{v}_{\mathbf{q}}=E_{\mathbf{q}, \mathbf{q}}$, which means that

$$
\mathbf{q}_{\mathbf{v}}=\left(E_{\mathbf{q}, \mathbf{q}}\right)^{-1} \text {. }
$$

Using this and the homogeneity (11) gives

$$
\begin{aligned}
E(\mathbf{q})_{t} & =\mathbf{v}^{T} \mathbf{q}_{t}=\mathbf{v}^{T}\left(E_{\mathbf{q}, \mathbf{q}}\right)^{-1} \mathbf{v}_{t}= \\
& \left(\mathbf{v}^{T}\left(E_{\mathbf{q}, \mathbf{q}}\right)^{-1} \mathbf{v}\right)_{t}-\left(\mathbf{v}^{T}\left(E_{\mathbf{q}, \mathbf{q}}\right)^{-1}\right)_{t} \mathbf{v}=\left(\mathbf{v}^{T}\left(E_{\mathbf{q}, \mathbf{q}}\right)^{-1} \mathbf{v}\right)_{t}-\beta \mathbf{q}_{t}^{T} \mathbf{v}
\end{aligned}
$$

so that

$$
(\beta+1) \mathbf{v}^{T} \mathbf{q}_{t}=\left(\mathbf{v}^{T}\left(E_{\mathbf{q}, \mathbf{q}}\right)^{-1} \mathbf{v}\right)_{t},
$$

which proves the Lemma.

With the help of Lemma 2, (19) can be written

$$
\frac{d}{d t}\left(\mathbf{v},\left(E_{\mathbf{q}, \mathbf{q}}\right)^{-1} \mathbf{v}\right)=-\beta\left(\mathbf{v}(b, t)^{T} \mathbf{f}(b, t)-\mathbf{v}(a, t)^{T} \mathbf{f}(a, t)\right)
$$

or, equivalently, by use of (13),

$$
\frac{d}{d t}\left(\mathbf{v},\left(E_{\mathbf{q}, \mathbf{q}}\right)^{-1} \mathbf{v}\right)=-\left(\mathbf{v}(b, t)^{T} \mathbf{f}_{\mathbf{v}} \mathbf{v}(b, t)-\mathbf{v}(a, t)^{T} \mathbf{f}_{\mathbf{v}} \mathbf{v}(a, t)\right) .
$$

Note that the left hand side is not an $L^{2}$-norm, since the matrix $E_{\mathbf{q}, \mathbf{q}}^{-1}$ depends on $\mathbf{v}$. It is necessary to bound the eigenvalues of $E_{\mathbf{q}, \mathbf{q}}^{-1}$ in order to make (22) a valid estimate. 


\section{Semi-discrete entropy split discretization of the Euler equations}

Consider the 1D compressible gas dynamic equations (16) discretized on a domain $a<x<b$ by a uniform grid $x_{j}=(j-1) \Delta x+a, j=1, \ldots, N$, and grid spacing $\Delta x=(b-a) /(N-1)$. Define the semi-discrete entropy split approximation

$$
\frac{d}{d t} \mathbf{q}_{j}+\frac{\beta}{\beta+1} D \mathbf{f}_{j}+\frac{1}{\beta+1}\left(\mathbf{f}_{\mathbf{v}}\right)_{j} D \mathbf{v}_{j}=0, \quad j=1, \ldots, N,
$$

where $D$ is a SBP difference operator. With entropy split scheme, we will always mean the entropy split form of the equations (17) discretized in space by a summation-by-parts finite difference operator. The flux Jacobian matrix with respect to the entropy variables, $\mathbf{f}_{\mathbf{v}}$, is symmetric. The SBP scalar product is denoted by

$$
(\mathbf{u}, \mathbf{v})_{h}=\Delta x \sum_{j=1}^{N} \omega_{j} \mathbf{u}_{j}^{T} \mathbf{v}_{j},
$$

where $\omega_{j}>0$ are weights that are different from 1 only at a few points near the boundaries. The operator $D$ satisfies the SBP property

$$
(D \mathbf{u}, \mathbf{v})_{h}=-(\mathbf{u}, D \mathbf{v})_{h}-\mathbf{u}_{1}^{T} \mathbf{v}_{1}+\mathbf{u}_{N}^{T} \mathbf{v}_{N},
$$

but is otherwise arbitrary. In the most common case $D$ is a standard SBP centered difference operator, but other operators are possible. For example, $D$ could be a bandwidth optimized operator with SBP closure such as developed in [11].

A zero velocity, $u_{1}=0, u_{N}=0$, boundary condition is enforced, corresponding to wall boundaries. Thanks to the SBP property of the difference approximation the derivation of entropy conservation for the continuous problem can be carried over to the discretization.

Theorem 1. The approximation (23) together with the boundary conditions $u_{1}=0$ and $u_{N}=0$ conserve the global entropy in the sense that

$$
\frac{d}{d t} \sum_{j=1}^{N} \omega_{j} E_{j}=0 .
$$

Proof. Denote

$$
\mathbf{r}=-\frac{\beta}{\beta+1}(\mathbf{v}, D \mathbf{f})_{h}-\frac{1}{\beta+1}\left(\mathbf{v},\left(\mathbf{f}_{\mathbf{v}}\right) D \mathbf{v}\right)_{h} .
$$

The scheme (23) can be written 


$$
\frac{d}{d t} \mathbf{q}_{j}=P \mathbf{r}_{j},
$$

where the projection $P$ sets $u_{1}=0$ and $u_{N}=0$. Because $P^{2}=P$, applying $\mathrm{P}$ to both sides of (25) gives that

$$
\frac{d}{d t} P \mathbf{q}=\frac{d}{d t} \mathbf{q}
$$

i.e., that $P \mathbf{q}=\mathbf{q}$ if the initial data satisfy the boundary conditions. For the entropy

$$
\begin{aligned}
\frac{d}{d t} E=\left(\mathbf{v}, \mathbf{q}_{t}\right)_{h}=(\mathbf{v}, P \mathbf{r})_{h}=(\mathbf{v}, \mathbf{r})_{h}-(\mathbf{v},(I-P) \mathbf{r})_{h}= \\
(\mathbf{v}, \mathbf{r})_{h}-(P \mathbf{v},(I-P) \mathbf{r})_{h}=(\mathbf{v}, \mathbf{r})_{h},
\end{aligned}
$$

where we use that $P \mathbf{v}=\mathbf{v}$, because the second component of $\mathbf{v}$ is zero when the $x$-velocity, $u$, is zero, and the orthogonality $(P \mathbf{v},(I-P) \mathbf{r})_{h}=0$. The entropy equation is now of the same form as for the continuous problem, and the same technique as used in (18), but with integration-by-parts replaced by summation-by-parts gives

$$
\frac{d}{d t} E\left(\mathbf{q}_{j}\right)=-F_{N}+F_{1} .
$$

Entropy conservation follows by observing that $F=u E$, so that the boundary conditions imply that $F_{1}=F_{N}=0$.

If the boundary conditions are periodic, no SBP modification of the difference operator is needed. Entropy conservation is proved with periodic boundary conditions by direct application of the same technique as above.

Remark The result in Lemma 2 carries over directly to the semi-discrete approximation, since only time derivatives are used in the proof. Hence, the $L^{2}$-like estimate

$$
\frac{d}{d t} \sum_{j=1}^{N} \omega_{j} \mathbf{v}_{j}\left(E_{\mathbf{q}, \mathbf{q}}\right)_{j}^{-1} \mathbf{v}_{j}=0
$$

is obtained for the approximation (23).

\section{Comparison between the entropy split scheme and Tadmor-type entropy conserving schemes}

This section reviews entropy conserving schemes on conservative form and discusses the differences between these schemes and the entropy split approximation (23). 
Entropy conserving schemes are written in conservative form

$$
\frac{d}{d t} \mathbf{q}_{j}(t)+\frac{1}{\Delta x}\left(\mathbf{h}_{j+1 / 2}^{E C}-\mathbf{h}_{j-1 / 2}^{E C}\right)=0 \quad j=1, \ldots, N,
$$

where $\mathbf{h}_{j+1 / 2}^{E C}$ are numerical flux functions. A second order accurate entropy conservative scheme was developed by E. Tadmor in the 1980s, using a two point numerical flux function,

$$
\mathbf{h}_{j+1 / 2}^{E C}=\mathbf{h}^{E C}\left(\mathbf{q}_{j+1}, \mathbf{q}_{j}\right) .
$$

Tadmor showed that if the flux is defined such that the property

$$
\left(\mathbf{v}_{j+1}-\mathbf{v}_{j}\right)^{T} \mathbf{h}^{E C}\left(\mathbf{q}_{j+1}, \mathbf{q}_{j}\right)=\psi_{j+1}-\psi_{j}
$$

holds, then (27) implies the local entropy conservation (6), and thereby also global entropy conservation (3); see [30]. Tadmor's scheme is second order accurate and is defined for periodic boundary conditions only. The entropy flux potential, $\psi$ in (28), is defined by

$$
\psi=\mathbf{v}^{T} \mathbf{f}-F .
$$

Generalization to arbitrary order was done in [14]. In fact, any centered approximation of the form

$$
D f_{j}=\frac{1}{\Delta x} \sum_{k=1}^{p} \alpha_{k}\left(f_{j+k}-f_{j-k}\right)
$$

can be used as a template for a corresponding entropy conserving scheme. This is done by writing

$$
D f_{j}=\frac{1}{\Delta x} \sum_{k=1}^{p} \alpha_{k}\left(f_{j+k}+f_{j}-\left(f_{j-k}+f_{j}\right)\right)
$$

and since $\left(f_{j+k}+f_{j}\right) / 2$ has the form of a second order centered flux, it is replaced by the second order accurate entropy conservative flux function to define the general entropy conservative scheme as,

$$
\frac{d}{d t} \mathbf{q}_{j}(t)+\frac{1}{\Delta x} \sum_{k=1}^{p} 2 \alpha_{k}\left(\mathbf{h}^{E C}\left(\mathbf{q}_{j+k}, \mathbf{q}_{j}\right)-\mathbf{h}^{E C}\left(\mathbf{q}_{j}, \mathbf{q}_{j-k}\right)\right)=0 .
$$

This is not explicitly in conservative form, but it is straightforward to rewrite (31) as a flux difference; see, e.g., [22].

In the case of periodic boundaries it is was shown in [14] (see also [28] and [22]) that the order of accuracy of (31) is the same as the accuracy of the linear operator (30), and that entropy conservation follows by using the 
property (28). The local entropy conservation (6) also holds for (31). For details see the cited references.

A boundary modification of the entropy conserving scheme (31) was developed in [4]. The boundary modification is designed such that the scheme satisfies entropy conservation with boundary fluxes (4). The boundary modification is based on exactly the same idea as used to define (31), but the template operator is a matrix where boundary operators are included. The general linear difference operator is a matrix $\Delta x D=\left(\alpha_{j k}\right)$, approximating the derivative $f_{x}$ at the point $j$,

$$
(D f)_{j}=\frac{1}{\Delta x} \sum_{k=1}^{N} \alpha_{j, k} f_{k}, \quad j=1, \ldots, N,
$$

where $\sum_{k=1}^{N} \alpha_{j, k}=0$, meaning that derivatives of constants are exactly zero. An entropy conserving scheme corresponding to this operator is defined by

$$
\frac{d}{d t} \mathbf{q}_{j}(t)+\frac{1}{\Delta x} \sum_{k=1}^{N} 2 \alpha_{j, k} \mathbf{h}^{E C}\left(\mathbf{q}_{k}, \mathbf{q}_{j}\right)=0, \quad j=1, \ldots, N .
$$

In the standard case $D$ is a fixed centered operator of the form (30) away from boundaries, and has SBP modified operators near the domain boundaries. For that case, the scheme (32) is identical to (31) away from boundaries.

It is straightforward to derive the discrete entropy conservation law

$$
\frac{d}{d t} E_{j}(t)+\frac{1}{\Delta x} \sum_{k=1}^{N} 2 \alpha_{j, k} H\left(\mathbf{q}_{k}, \mathbf{q}_{j}\right)=0, \quad j=1, \ldots, N
$$

from (32). The numerical entropy flux is defined by

$$
H\left(\mathbf{q}_{k}, \mathbf{q}_{j}\right)=-\frac{1}{2}\left(\psi_{j}+\psi_{k}\right)+\frac{1}{2}\left(\mathbf{v}_{j}+\mathbf{v}_{k}\right)^{T} \mathbf{h}^{E C}\left(\mathbf{q}_{k}, \mathbf{q}_{j}\right),
$$

which is consistent with the entropy flux,

$$
H(\mathbf{q}, \mathbf{q})=\psi+\mathbf{v}^{T} \mathbf{h}(\mathbf{q}, \mathbf{q})=\psi+\mathbf{v}^{T} \mathbf{f}(\mathbf{q})=F(\mathbf{q}) .
$$

To derive (33), multiply (32) by $\mathbf{v}_{j}$, and rewrite 


$$
\begin{aligned}
& \sum_{k} \alpha_{j, k} \mathbf{v}_{j}^{T} \mathbf{h}^{E C}\left(\mathbf{q}_{k}, \mathbf{q}_{j}\right) \\
&=\sum_{k} \alpha_{j, k}\left(\frac{1}{2}\left(\mathbf{v}_{j}-\mathbf{v}_{k}\right)^{T} \mathbf{h}^{E C}\left(\mathbf{q}_{k}, \mathbf{q}_{j}\right)+\frac{1}{2}\left(\mathbf{v}_{j}+\mathbf{v}_{k}\right)^{T} \mathbf{h}^{E C}\left(\mathbf{q}_{k}, \mathbf{q}_{j}\right)\right) \\
&=\sum_{k} \alpha_{j, k}\left(\frac{1}{2}\left(\psi_{j}-\psi_{k}\right)+\frac{1}{2}\left(\mathbf{v}_{j}+\mathbf{v}_{k}\right)^{T} \mathbf{h}^{E C}\left(\mathbf{q}_{k}, \mathbf{q}_{j}\right)\right) \\
&=\sum_{k} \alpha_{j, k}\left(-\frac{1}{2}\left(\psi_{j}+\psi_{k}\right)+\frac{1}{2}\left(\mathbf{v}_{j}+\mathbf{v}_{k}\right)^{T} \mathbf{h}^{E C}\left(\mathbf{q}_{k}, \mathbf{q}_{j}\right)\right),
\end{aligned}
$$

where the last equality follows from $\sum_{k} \alpha_{j, k}=0$. By making the definition (34), (33) follows. The discrete equations (32) and (33) are not explicitly in conservative form, but their spatial derivatives are straightforward to rewrite as numerical flux differences.

The following theorem summarizes results from [4] and [17].

Theorem 2. Let $D=\left(\alpha_{j, k}\right)$ be a SBP operator, i.e., satsifying (24), and let $\mathbf{h}^{E C}$ be a second order accurate two point flux satisfying (28). Then the scheme (32) satisfies global entropy conservation with boundary fluxes (4). Moreover, the order of accuracy of (32) is the same as the order of accuracy of $D$.

Next we give a proof of the entropy conservation property using a different notation from [4].

The global conservation (4) follows by using the SBP property (24). In matrix form (24) can be written

$$
W D=-D^{T} W+B,
$$

where $W$ is the diagonal matrix of norm weights, $W=\Delta x \operatorname{diag}\left(\omega_{1}, \omega_{2}, \ldots, \omega_{N}\right)$, and $D$ is the SBP operator, and $B$ is the matrix of boundary terms, $B_{1,1}=-1, B_{N, N}=1$. The remaining elements of $B$ are zero. Define the matrix scalar product between two $N \times N$ matrices,

$$
A \odot B=\sum_{i=1}^{N} \sum_{j=1}^{N} a_{i, j} b_{i, j} .
$$

Then, for any $N \times N$ matrix $X(36)$ gives

$$
(W D) \odot X=-\left(D^{T} W\right) \odot X+B \odot X,
$$

which, since $A^{T} \odot B^{T}=A \odot B$, can be written

$$
(W D) \odot X=-(W D) \odot X^{T}+B \odot X .
$$

We will call (37) generalized summation-by-parts identity, since it generalizes (24) in the sense that (37) becomes (24) for the special case when $X$ is the 
rank-1 matrix $u v^{T}$ for grid functions $u$ and $v$. With the help of (37) we prove that (33) implies the global conservation (4). Multiplying (33) by the norm weights, $\omega_{j}$, and taking the sum over $j$, gives

$$
\Delta x \frac{d}{d t} \sum_{j=1}^{N} \omega_{j} E_{j}(t)+\sum_{j=1}^{N} \sum_{k=1}^{N} 2 \omega_{j} \alpha_{j, k} H\left(\mathbf{q}_{k}, \mathbf{q}_{j}\right)=0 .
$$

The flux term can be expressed as

$$
2(W D) \odot X
$$

with $X_{j, k}=H\left(\mathbf{q}_{k}, \mathbf{q}_{j}\right)$. It follows from the definition of $H,(34)$, that $X$ is a symmetric matrix, and hence (37) gives that

$$
2(W D) \odot X=B \odot X=H\left(\mathbf{q}_{N}, \mathbf{q}_{N}\right)-H\left(\mathbf{q}_{1}, \mathbf{q}_{1}\right)=F_{N}-F_{1},
$$

and

$$
\Delta x \frac{d}{d t} \sum_{j=1}^{N} \omega_{j} E_{j}(t)+F_{N}-F_{1}=0
$$

follows.

Remark The entropy conservation proof above carries through also in the case full norms, i.e., where $W$ is symmetric positive definite, but not necessarily diagonal.

Table 1 compares the entropy split scheme (23) and the entropy conservative scheme in conservation form. Both schemes (23) and (32) satisfy the same properties. The entropy split scheme is simpler to define, since it does not require any second order accurate flux $\mathbf{h}^{E C}$. The entropy conservative scheme can be written in conservation form, which is advantageous when shocks are present. However, since these schemes have no built-in dissipation, they are not suited for shock wave computation.

\begin{tabular}{|l|l|l|}
\hline & Entropy split (23) & Entropy cons. (32) \\
\hline Requires & SBP op. $D$ & SBP op. $D$ and 2nd order EC flux $\mathbf{h}^{E C}$ \\
Satisfies & $(4)$ & $(4)$ \\
Conservation form & No & Yes \\
Order of accuracy & Given by $D$ & Given by $D$ \\
Entropy & Requires $(9)$ & (38) or $(9)$ (shown below) \\
\hline
\end{tabular}

Table 1 Comparison between high order entropy split and high order Tadmor-type entropy conservation schemes.

The exact construction of the second order accurate EC numerical flux, which (32) requires, is dependent on the entropy used. For the Euler equations there are two entropies that are considered. 
1. The logarithmic entropy

$$
E=-\rho \log \left(p \rho^{-\gamma}\right)
$$

2. Harten's entropies (9), which is a family of entropies, depending on a parameter, $\alpha$.

Most numerical fluxes, $\mathbf{h}^{E C}$, published to date use the logarithmic entropy (38); see $[10,35,25]$. It will be shown in the next section that construction of $\mathbf{h}^{E C}$ is also possible for the entropies (9).

\section{Tadmor-type Entropy Conservative Discretization using the Harten entropy}

This section derives an entropy conservative scheme for the entropy (9), by a technique similar to the one used to derive an Tadmor-type of entropy conservative scheme for the logarithmic entropy in [35] for both the gas dynamics and MHD.

The approximation will be in conservative form. In the one-dimensional case, for example,

$$
\frac{d}{d t} \mathbf{q}_{j}+\frac{1}{\Delta x}\left(\mathbf{h}_{j+1 / 2}-\mathbf{h}_{j-1 / 2}\right)=\mathbf{0 .} \quad j=1, \ldots, N
$$

will approximate (16). The numerical flux function, $\mathbf{h}_{j+1 / 2}=\mathbf{h}\left(\mathbf{q}_{j+1}, \mathbf{q}_{j}\right)$, satisfies

$$
\left(\mathbf{v}_{j+1}-\mathbf{v}_{j}\right)^{T} \mathbf{h}_{j+1 / 2}=\psi_{j+1}-\psi_{j}
$$

where $\psi$ is the entropy flux potential,

$$
\psi=\mathbf{v}^{T} \hat{\mathbf{f}}-\hat{F}=s^{\frac{1}{\alpha+\gamma}} \rho \hat{u},
$$

and where $\mathbf{v}$ denotes the entropy variables (10). The numerical flux $\mathbf{h}_{j+1 / 2}$ approximates the flux $\hat{\mathbf{f}}=k_{1} \mathbf{f}+k_{2} \mathbf{g}+k_{3} \mathbf{h}$. Forward differencing is denoted by $\Delta u=\left(u_{j+1}-u_{j}\right)$. It is well-known that (40) implies entropy conservation of the corresponding semi-discrete numerical scheme; see [30].

In order to derive an entropy conservative scheme, we define the parameter vector

$$
\mathbf{z}=\left(\frac{\rho}{p}\left(p \rho^{-\gamma}\right)^{\frac{1}{\alpha+\gamma}} u v w p\right)^{T} .
$$

This definition leads to the following expressions for the entropy variables (10) and entropy flux potential in terms of the components of $\mathbf{z}$, 
Title Suppressed Due to Excessive Length

$$
\begin{aligned}
v_{1} & =-\frac{1}{2} z_{1}\left(z_{2}^{2}+z_{3}^{2}+z_{4}^{2}\right)-\frac{\alpha}{\gamma-1} z_{1}^{-\frac{\gamma}{\alpha}} z_{5}^{-\frac{\gamma-1}{\alpha}} \\
v_{2} & =z_{1} z_{2} \quad v_{3}=z_{1} z_{3} \quad v_{4}=z_{1} z_{4} \\
v_{5} & =-z_{1} \\
\psi & =z_{1} z_{5} \hat{z}
\end{aligned}
$$

where $\hat{z}=k_{1} z_{2}+k_{2} z_{3}+k_{3} z_{4}$.

The average will be denoted by

$$
\{u\}=\left(u_{j+1}+u_{j}\right) / 2 \text {, }
$$

and the exponential average will be denoted by

$$
\left\{u^{\beta-1}\right\}_{e}=\frac{1}{\beta} \frac{u_{j+1}^{\beta}-u_{j}^{\beta}}{u_{j+1}-u_{j}} .
$$

Note that $\left\{u^{\beta-1}\right\}_{e}$ can be considered an average, since the right hand side expression approximates $u^{\beta-1}$ when $\left|u_{j+1}-u_{j}\right|$ is small.

Remark To evaluate (41) when $\left|u_{j+1}-u_{j}\right|$ is close to zero we rewrite

$$
\frac{1}{\beta} \frac{u_{j+1}^{\beta}-u_{j}^{\beta}}{u_{j+1}-u_{j}}=u_{j}^{\beta-1} \frac{1}{\beta} \frac{r^{\beta}-1}{r-1}
$$

where $r=u_{j+1} / u_{j}$, and approximate

$$
\frac{1}{\beta} \frac{r^{\beta}-1}{r-1} \approx 1+\frac{1}{2}(\beta-1)(r-1)+\frac{1}{6}(\beta-1)(\beta-2)(r-1)^{2}
$$

for $|r-1|<10^{-4}$. This will give a relative error $\approx 10^{-12}$.

For the derivation of the entropy conserving numerical fluxes, the following formulas are used.

$$
\begin{aligned}
\Delta v_{1}= & -\left\{z_{1}\right\}\left(\left\{z_{2}\right\} \Delta z_{2}+\left\{z_{3}\right\} \Delta z_{3}+\left\{z_{4}\right\} \Delta z_{4}\right)-\frac{1}{2}\left\{z_{2}^{2}+z_{3}^{2}+z_{4}^{2}\right\} \Delta z_{1} \\
& -\frac{\alpha}{\gamma-1}\left\{z_{1}^{-\gamma / \alpha}\right\} \Delta z_{5}^{-(\gamma-1) \alpha}-\frac{\alpha}{\gamma-1}\left\{z_{5}^{-(\gamma-1) \alpha}\right\} \Delta z_{1}^{-\gamma / \alpha} \\
= & -\left\{z_{1}\right\}\left(\left\{z_{2}\right\} \Delta z_{2}+\left\{z_{3}\right\} \Delta z_{3}+\left\{z_{4}\right\} \Delta z_{4}\right)-\frac{1}{2}\left\{z_{2}^{2}+z_{3}^{2}+z_{4}^{2}\right\} \Delta z_{1} \\
- & \left\{z_{1}^{-\gamma / \alpha}\right\}\left\{z_{5}^{-(\gamma+\alpha-1) / \alpha}\right\}_{e} \Delta z_{5}-\frac{\gamma}{\gamma-1}\left\{z_{5}^{-(\gamma-1) \alpha}\right\}\left\{z_{1}^{-(\gamma+\alpha) / \alpha}\right\}_{e} \Delta z_{1}
\end{aligned}
$$

and 


$$
\begin{aligned}
& \Delta v_{2}=\left\{z_{2}\right\} \Delta z_{1}+\left\{z_{1}\right\} \Delta z_{2}, \quad \Delta v_{3}=\left\{z_{3}\right\} \Delta z_{1}+\left\{z_{1}\right\} \Delta z_{3}, \\
& \Delta v_{4}=\left\{z_{4}\right\} \Delta z_{1}+\left\{z_{1}\right\} \Delta z_{4}, \quad \Delta v_{5}=-\Delta z_{5}, \\
& \Delta \psi=\left\{z_{1} \hat{z}\right\} \Delta z_{5}+\left\{z_{1}\right\}\left\{z_{5}\right\} \Delta \hat{z}+\left\{z_{5}\right\}\{\hat{z}\} \Delta z_{1} .
\end{aligned}
$$

Inserting this into (40) and solving for the numerical fluxes give

$$
\begin{aligned}
h_{1} & =\frac{\left\{z_{1} \hat{z}\right\}}{\left\{z_{1}^{-\gamma / \alpha}\right\}\left\{z_{5}^{(1-\gamma-\alpha) / \alpha}\right\}_{e}} \\
h_{2} & =\left\{z_{2}\right\} h_{1}+k_{1}\left\{z_{5}\right\} \\
h_{3} & =\left\{z_{3}\right\} h_{1}+k_{2}\left\{z_{5}\right\} \\
h_{4} & =\left\{z_{4}\right\} h_{1}+k_{3}\left\{z_{5}\right\}
\end{aligned}
$$

with energy flux

$$
\begin{array}{r}
h_{5}=h_{1}\left(\frac{\gamma}{\gamma-1}\left\{z_{5}^{-(\gamma-1) / \alpha}\right\}\left\{z_{1}^{-(\gamma+\alpha) / \alpha}\right\}_{e}+\left\{z_{2}\right\}^{2}+\left\{z_{3}\right\}^{2}+\left\{z_{4}\right\}^{2}-\right. \\
\left.\frac{1}{2}\left\{z_{2}^{2}+z_{3}^{2}+z_{4}^{2}\right\}\right) .
\end{array}
$$

Rewritten in terms of physical variables (and $z_{1}$ ) the numerical fluxes become

$$
\begin{aligned}
h_{1} & \left.=\frac{\left\{z_{1} \hat{u}\right\}}{\left\{z_{1}^{-\gamma / \alpha}\right\}\left\{\rho^{-1} z_{1}^{1+\frac{\gamma}{\alpha}}\right\}_{e}}\right) \\
h_{2} & =h_{1}\{u\}+k_{1}\{p\} \\
h_{3} & =h_{1}\{v\}+k_{2}\{p\} \\
h_{4} & =h_{1}\{w\}+k_{3}\{p\}
\end{aligned}
$$

and

$$
\begin{aligned}
h_{5}=h_{1}\left(\frac{\gamma}{\gamma-1}\left\{p^{-(\gamma-1) / \alpha}\right\}\right. & \left\{\rho^{-1} p^{1+\frac{\gamma-1}{\alpha}}\right\}_{e} \\
+ & \left.\{u\}^{2}+\{v\}^{2}+\{w\}^{2}-\frac{1}{2}\left\{u^{2}+v^{2}+w^{2}\right\}\right) .
\end{aligned}
$$

In summary, the numerical flux function $\mathbf{h}_{j+1 / 2}$ formed by the five vector components (43)-(47) gives an entropy conserving numerical flux that conserves the entropy (9) when used in a semi-discrete scheme. For the one dimensional case (39) the direction vector is $\left(\begin{array}{lll}k_{1} & k_{2} & k_{3}\end{array}\right)=\left(\begin{array}{lll}1 & 0 & 0\end{array}\right)$.

As can be seen, the derivation using the entropies (9) to construct entropy conserving schemes follows naturally the steps in [25]. It is about twice more CPU intensive than the entropy split scheme for the same central spatial discretization. 


\section{Treating flows with discontinuities}

A scheme with conservation of entropy is not sufficient to obtain a physically relevant solution when shock waves are present. In order to compute flows with shock waves, entropy conserving schemes must be augmented with numerical dissipation that decreases the entropy across shock waves. We next review some existing methods to combine entropy conservation with shock capturing schemes. See [1] for an overview and a discusion of the entropy viscosity method for nonlinear conservation equations.

One approach to shock wave computation is to define a shock capturing method directly in entropy variables. For example, Ismail and Roe in [10] define a Roe-type Riemann solver in entropy variables. Similarly, in [5] an ENO scheme is defined in entropy variables. It would have been possible to add dissipation defined in the entropy variables directly to the entropy split scheme (23) to define a shock capturing variant of the scheme with guaranteed entropy dissipation. This is outside the scope of the current article, but an interesting subject for future work.

Another approach to shock wave computing with entropy dissipation is taken in [4], where a WENO scheme is used together with an entropy limiter. We here review how this is done. By a simple modification of the results in Section 6 , one can show that if the relation (28) holds with inequality,

$$
\left(\mathbf{v}_{j+1}-\mathbf{v}_{j}\right)^{T} \mathbf{h}^{E C}\left(\mathbf{q}_{j+1}, \mathbf{q}_{j}\right) \geq \psi_{j+1}-\psi_{j}
$$

then the entropy inequality (5) follows. This is used in [4] for flux comparison. It follows from equations (28) and (48) that if

$$
\left(\mathbf{v}_{j+1}-\mathbf{v}_{j}\right)^{T}\left(\mathbf{h}_{j+1 / 2}^{e c}-\mathbf{h}_{j+1 / 2}\right) \leq 0
$$

with $\mathbf{h}_{j+1 / 2}^{e c}$ the numerical flux of an entropy conserving scheme, then the entropy computed using the flux $\mathbf{h}_{j+1 / 2}$ will decrease. Whenever (49) is not satisfied by the WENO flux, the weight, $w_{j+1 / 2}$, in the hybrid flux

$$
\mathbf{h}_{j+1 / 2}^{E C W E N O}=w_{j+1 / 2} \mathbf{h}_{j+1 / 2}^{e c}-\left(1-w_{j+1 / 2}\right) \mathbf{h}_{j+1 / 2}^{W E N O}
$$

is determined such that (49) holds, and the hybrid flux $\mathbf{h}_{j+1 / 2}^{E C W E N O}$ is used instead of the WENO flux $\mathbf{h}_{j+1 / 2}^{W E N O}$. Obviously, there always exists such a weight, since taking $w_{j+1 / 2}=1$ gives entropy conservation. See [4] for the exact formula for $w_{j+1 / 2}$.

Our treatment for flows containing discontinuities by the entropy split scheme is by the Yee et al. nonlinear filter approach $[36,37,38,39,25,24]$. The nonlinear filter approach consists of the dissipative portion of one's favorite high order shock-capturing method. After each full time step of the entropy split method, at each grid point a local flow sensor is employed to 
analyze the regularity of the computed flow data. Only strong discontinuity locations would receive the full amount of shock-capturing dissipation. In smooth regions no shock-capturing dissipation would be added, unless high frequency oscillations are developed due to the possibility of numerical instabilities from long time integration of the nonlinear governing equations. In regions with strong turbulence, if needed, a small fraction of the shockcapturing dissipation would be added to improve stability. These nonlinear filter approaches require one Riemann solver per time step per grid point for each spatial direction, independent of time discretization used in the entropy split scheme step.

The numerical experiments for shock wave computation here are to filter the computed solution after each time step with the dissipative portion of high order WENO scheme. The nonlinear filter can be applied together with either entropy split or entropy conserving methods on conservative form. The dissipation operator by itself is not guaranteed to decrease the entropy if a standard WENO dissipation is used. However, the dissipation is multiplied by a sensor that detects discontinuities and switches off the dissipation away from discontinuities. In this way the entropy conservation still holds locally where the solution is smooth. At the same time the WENO dissipation is active at discontinuities. We conjecture that it decreases the entropy at shock waves. Because the filter is applied after each completion of a full time step, the nonlinear filter approach cannot be written as a semi-discrete approximation. This makes a complete entropy analysis more difficult, since existing results from semi-discrete methods can not be used directly.

Due to our adaptive flow sensor to control the location and amount of numerical dissipation where needed, the dissipative portion of the high order shock-capturing scheme is only utilized at isolated computational regions, while maintaining high accuracy and entropy conservation/stability almost everywhere else. Our numerical experiments only considered less the CPU intensive dissipative portion of high resolution shock-capturing schemes as candidates for our nonlinear filter approach. Although the dissipative portion of optimized high order WENO, ENO, compact WENO, or another more CPU intensive high-resolution shocking-capturing scheme fits in the framework of our nonlinear filter scheme framework, due to the CPU intensive nature of these schemes, they are not considered in our numerical experiments. In addition, shock-capturing schemes that concentrate on capturing discontinuities with a narrowest grid stencil are usually designed for rapidly developing flows. Often, for a long time integration of DNS and LES type of flows, other added mechanisms are needed to improve nonlinear stability. 


\section{Numerical Experiments}

Previous studies using SBP boundary closures for non-periodic boundary conditions can be found in [37]. Here three standard long time integration test cases for a 2D smooth flow and two DNS computations are presented.

\subsection{Test Case 1: 2D Compressible Euler Simulation of Smooth Flow: Isentropic Vortex Convection}

The compressible Euler equations in two space dimensions are solved with initial data

$$
\begin{aligned}
& \rho(x, y)=\left(1-\frac{(\gamma-1) \beta^{2}}{8 \gamma \pi^{2}} e^{1-r^{2}}\right)^{\frac{1}{\gamma-1}} \\
& u(x, y)=u_{\infty}-\frac{\beta\left(y-y_{0}\right)}{2 \pi} e^{\left(1-r^{2}\right) / 2} \\
& v(x, y)=v_{\infty}+\frac{\beta\left(x-x_{0}\right)}{2 \pi} e^{\left(1-r^{2}\right) / 2} \\
& p(x, y)=\rho(x, y)^{\gamma},
\end{aligned}
$$

where $r^{2}=x^{2}+y^{2}, \beta=5, \gamma=1.4, u_{\infty}=1$, and $v_{\infty}=0$. The exact solution is the initial data translated, $\mathbf{u}(x, t)=\mathbf{u}_{0}\left(x-u_{\infty} t, y-v_{\infty} t\right)$.

The computational domain is $0 \leq x \leq 18,0 \leq y \leq 18$ with periodic boundary conditions. The center of the vortex is chosen to be $\left(x_{0}, y_{0}\right)=$ $(9,9)$. The problem is solved in time with the classical fourth-order accurate explicit Runge-Kutta method (RK4) to time $t=72$, which corresponds to four revolutions of the vortex across the domain.

Comparisons of high order classical central split schemes with high order DRP schemes with grid refinements are reported in [23]. Three levels of grid refinement were perform. Only one grid with maximum and $L_{2}$ error norms is compared with the exact solution shown in Fig. 1. Here C08-DS represents eighth-order central differencing applied to the Ducros et al. splitting form of the Euler flux derivatives. The corresponding eighth-order entropy splitting, entropy conservative method and Kennedy \& Grubber splitting are indicated by "C08-ES", "C08-EC" and "C08-KGS". If the computed solutions by "C08-DS", "C08-ES", "C08-EC" and "C08-KGS" are nonlinearly filtered by a dissipative portion of WENO7 (seventh-order weighted essentially nonoscillatory spatial method) with an adaptive flow sensor, they are indicated by C08-DS+WENO7FI, C08-ES+WENO7FI, C08-EC+WENO7FI, and C08-KGS+WENO7FI [36, 37, 38, 39, 25, 24]. For this smooth flow without any turbulent structure, $\beta=1$ for the entropy split scheme. The $\beta$ parameter studies are reported in $[37,19]$. In general, for compressible shockfree turbulence and turbulence with shocklets, $\beta$ lies somewhere in the range 
$1.5<\beta<2.5$. In general, the optimal $\beta$ is problem dependent. A general conclusion is that $\beta$ should not be very large or very small.

Other high resolution dissipative shock-capturing methods are also candidates for the nonlinear filter approach as well as other optimal WENO or ENO methods. However, with good control of the numerical dissipation away from discontinuities, there is no need to use the more complicated and more CPU intensive shock-capturing methods.

The non-split $\mathrm{C} 08$ without any added numerical dissipation diverges shortly after time evolution. Results by WENO5 or WENO7 are very diffusive with large maximum or $L_{2}$ errors.
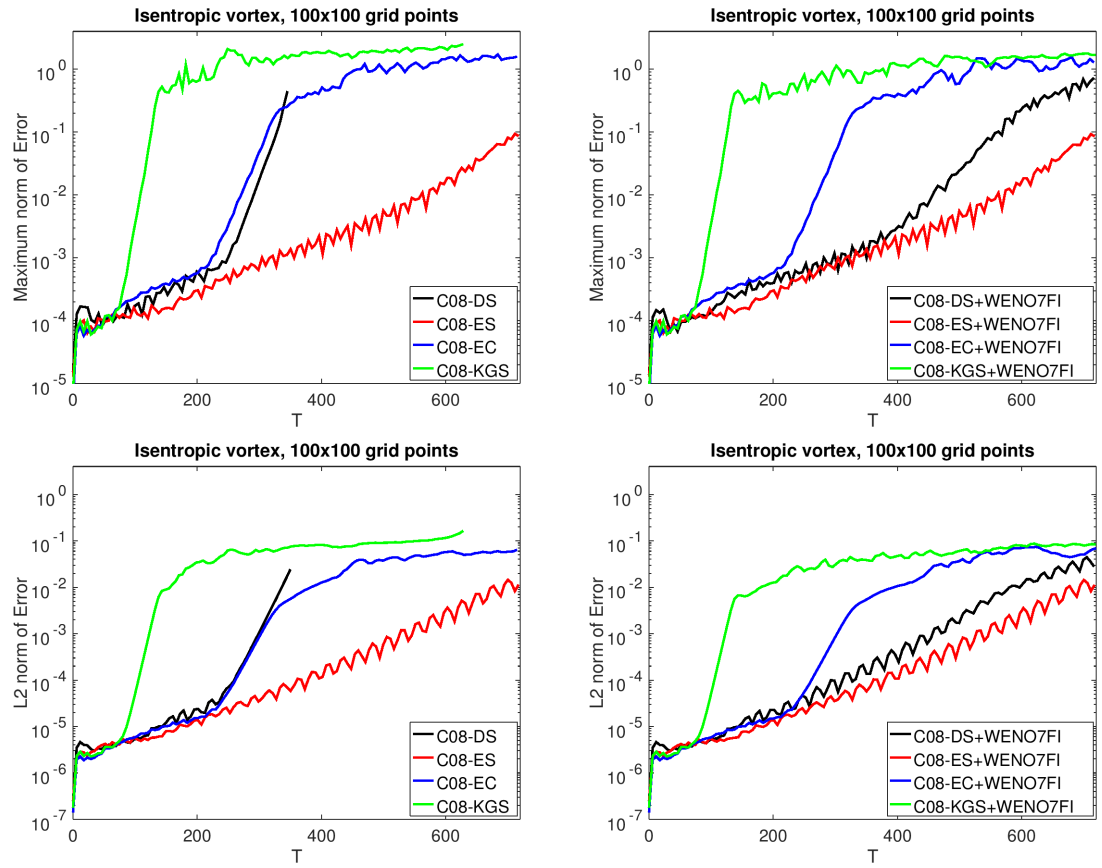

Fig. 1 Inviscid 2D compressible vortex convection with $100^{2}$ grid points: Comparison of maximum-norm of error vs. time for C08-DS, C08-ES, C08-EC, and C08-KGS (left, top), and C08-DS+WENO7FI, C08-ES+WENO7FI, C08-EC+WENO7FI, and C08KGS+WENO7fFI (right top). Bottom left and bottom right are the corresponding $L_{2}$-norm of error vs. time.

For this smooth flow integrated over a long time to observe nonlinear stability and accuracy among the four methods, the entropy split scheme is the most accurate method. 
Execution times for the base schemes used in the numerical experiments for the $100 \times 100$ grid were measured. The eighth-order schemes were run for 50 time steps on a single core of an Intel i7 processor. Times shown in the table are normalized with the time of C08-DS, which gave the fastest execution. In general, execution times depend on exactly how the code is written, on compiler and compiler settings used, and on the properties of the hardware. Using our non-optimized 3D code, the results are presented in Table 9.1 below.

The results in Table 9.1 is just one example of performance.

\begin{tabular}{lc}
\hline Method & Relative execution time \\
\hline C08-DS & 1.00 \\
C08-ES & 1.43 \\
C08-EC & 2.80 \\
C08-KGS & 1.50 \\
\hline
\end{tabular}

Table 2 Execution times for base schemes.

\subsection{Test Case 2: Inviscid 3D Taylor-Green Vortex}

The Taylor-Green vortex [32] is a well-known shock-free compressible turbulence test problem that has been studied extensively. The 3D Euler equations of compressible gas dynamics are solved with $\gamma=5 / 3$. The computational domain is a cube with sides of length $2 \pi$ and with periodic boundary conditions in all three directions. The initial data are

$$
\begin{aligned}
& \rho=1 \quad p=100+\{[\cos (2 z)+2)(\cos (2 x)+\cos (2 y)]-2\} / 16 \\
& u=\sin x \cos y \cos z, \quad v=-\cos x \sin y \cos z, \quad w=0 .
\end{aligned}
$$

The problem is solved to time 10 using a uniform grid of $64^{3}$ points. The solution develops smaller scales as time evolves. The increase of enstrophy with time, as seen in Fig. 2 (left subplot), is an indication of the growth rate of small scales. The figure compares results by the eight-order accurate entropy split scheme (23) (generalized to three space dimensions), denoted by C08ES, for values of the entropy parameter from $\beta=0.5$ to $\beta=4.5$ in steps of 0.5 . Figure 2 shows that the choice of $\beta$ has a large effect on the stability of the scheme. Out of the values tried, $\beta=2$ gives the smallest enstropy growth. The time integration was made by RK4, using CFL number 0.85 . Figure 3 shows the enstropy evolution with a larger range on the $y$ axis. The computation breaks down and does not reach the final time for $\beta=0.5,1.0,4.0$, and 4.5. The values $\beta=1.5,2.0,2.5,3.0$ and 3.5 all produce a bounded solution up to the final time. The evolution of the kinetic energy, 

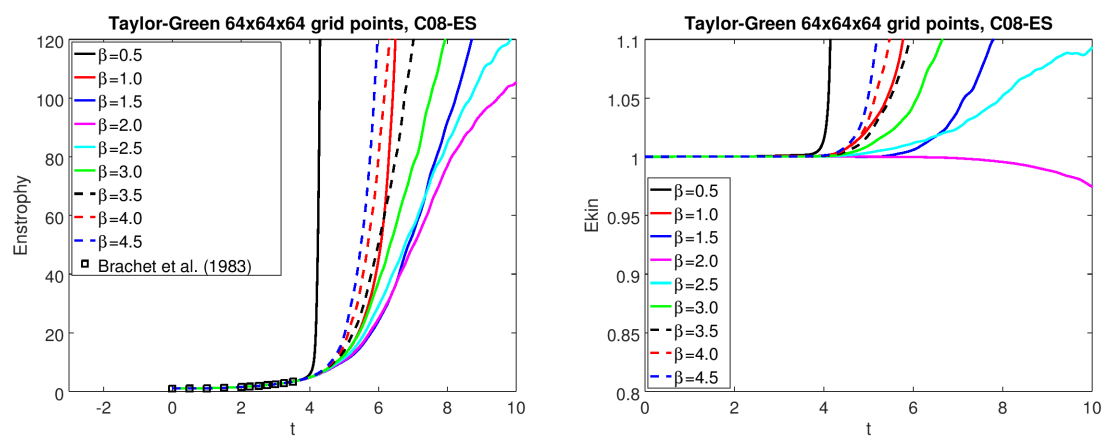

Fig. 2 3D Taylor-Green vortex with $64^{3}$ grid points. Evolution of enstrophy (left) and kinetic energy (right), for different values of parameter $\beta$. Eighth-order discretization.

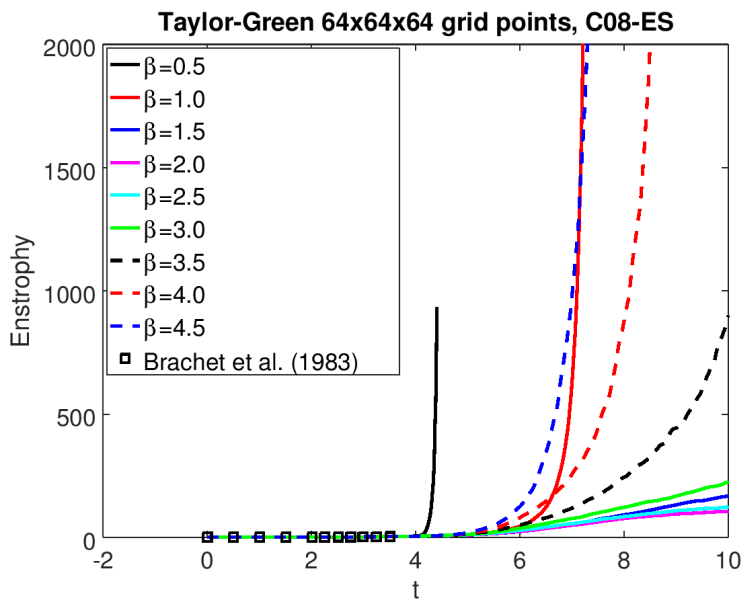

Fig. 3 3D Taylor-Green vortex: Enstropy vs. time, same as left subfigure of Fig. 2 but with larger range on the $y$-axis.

shown in the right subplot of Fig. 2, shows that of the values tried, only $\beta=2$ gives a decreasing kinetic energy. All other values lead to some growth.

Figure 4 shows the same computations as Fig. 2, but with spatial discretization by the tenth-order accurate entropy split scheme. The enstrophy increases somewhat faster for 10th-order discretization compared with the eight-order results in Fig. 2. This can be seen from detailed study of the intersection between the enstrophy curves and the line $t=10$ or $y=120$.

Figure 5 compares the enstrophy (left subplot) and kinetic energy (right subplot) computed by the non-linear filter schemes, where the base scheme is either entropy split, using $\beta=2$, and order of accuracy eight (C08-ES) or ten (C10-ES), or an entropy conserving method of order of accuracy eight (C08EC) or ten (C10-EC). The entropy conservative method is in conservation 

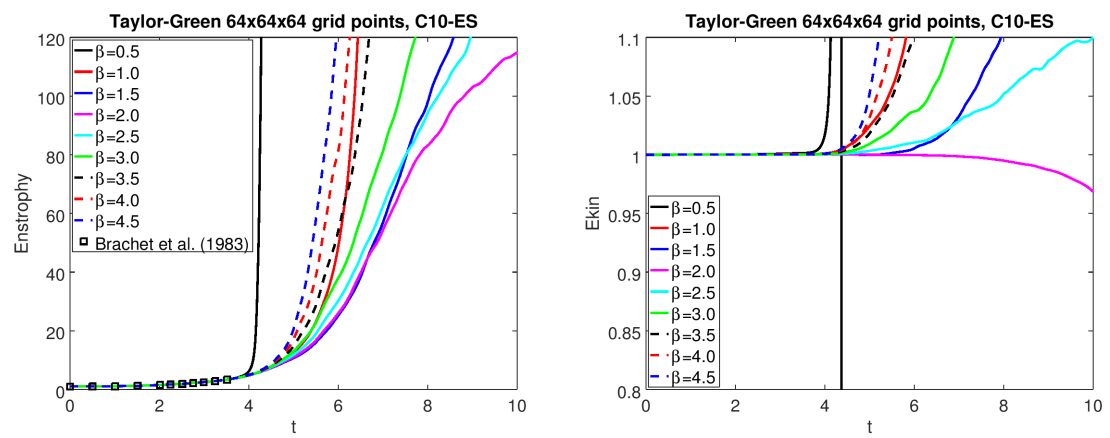

Fig. 4 3D Taylor-Green vortex with $64^{3}$ grid points: Evaluation of enstrophy (left) and kinetic energy (right), for different values of parameter $\beta$. Tenth-order discretization.

form and uses the entropy $-\frac{\rho}{\gamma-1} \ln \left(p \rho^{-\gamma}\right)$; see [25] for a detailed description. The nonlinear filter uses dissipation from the seventh-order WENO method (WENO7FI) together with eighth-order base schemes, and dissipation from the ninth-order WENO method (WENO9FI) together with tenth-order base schemes. The exact kinetic energy "Ekin" solution for this flow is unity.
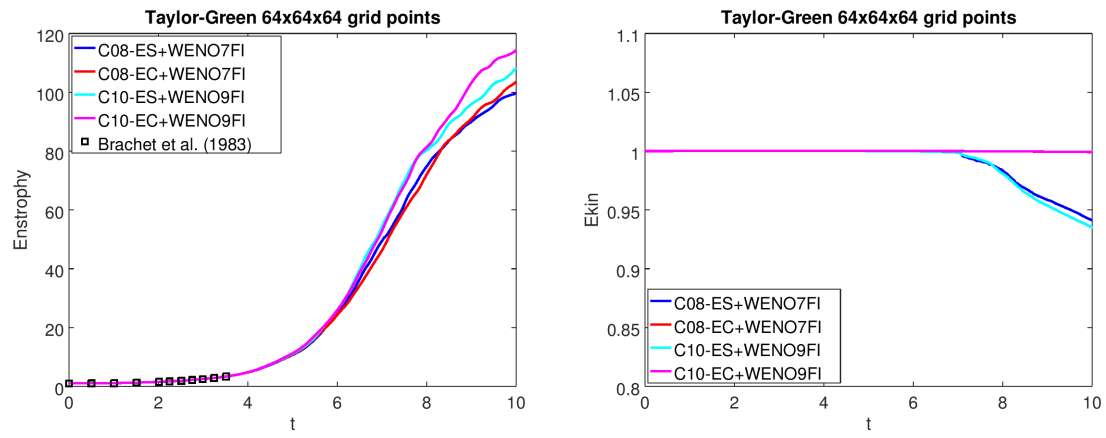

Fig. 5 3D Taylor-Green vortex with $64^{3}$ grid points: Evolution of enstrophy (left) and kinetic energy (right). Methods are C08-ES+WENO7FI (blue), C08-EC+WENO7FI (red), C10-ES+WENO9FI (cyan), and C10-EC+WENO9FI (magenta).

\subsection{Test Case 3: 3D Isotropic Turbulence with Eddy Shocklets}

The third numerical test problem computes decaying compressible isotropic turbulence with eddy shocklets. For high enough turbulent Mach numbers, 
weak shocks (shocklets) develop from the turbulent motion. In this test the initial turbulent Mach number is 0.6. The Navier-Stokes equations are solved using $\gamma=1.4$. The computational domain is a cube with side length $2 \pi$ and periodic boundary conditions in all three directions. The initial datum is a random divergent free velocity field, $u_{i, 0}, i=1,2,3$, that satisfies

$$
\frac{3}{2} u_{r m s, 0}^{2}=\frac{1}{2}\left\langle u_{i, 0}, u_{i, 0}\right\rangle=\int_{0}^{\infty} E(k) d k,
$$

with energy spectrum

$$
E(k) \sim k^{4} e^{-2\left(k / k_{0}\right)^{2}} .
$$

The computations were made with $u_{r m s, 0}=1$ and $k_{0}=4$. The angular brackets denote averaging over the entire computational domain. The density and pressure fields are initially constant. The Taylor-scale Reynolds number, $R e_{\lambda, 0}$, is 100. See [13] for definitions of the quantities and more details about the set up of the problem. The simulation is run to the final time 4 .
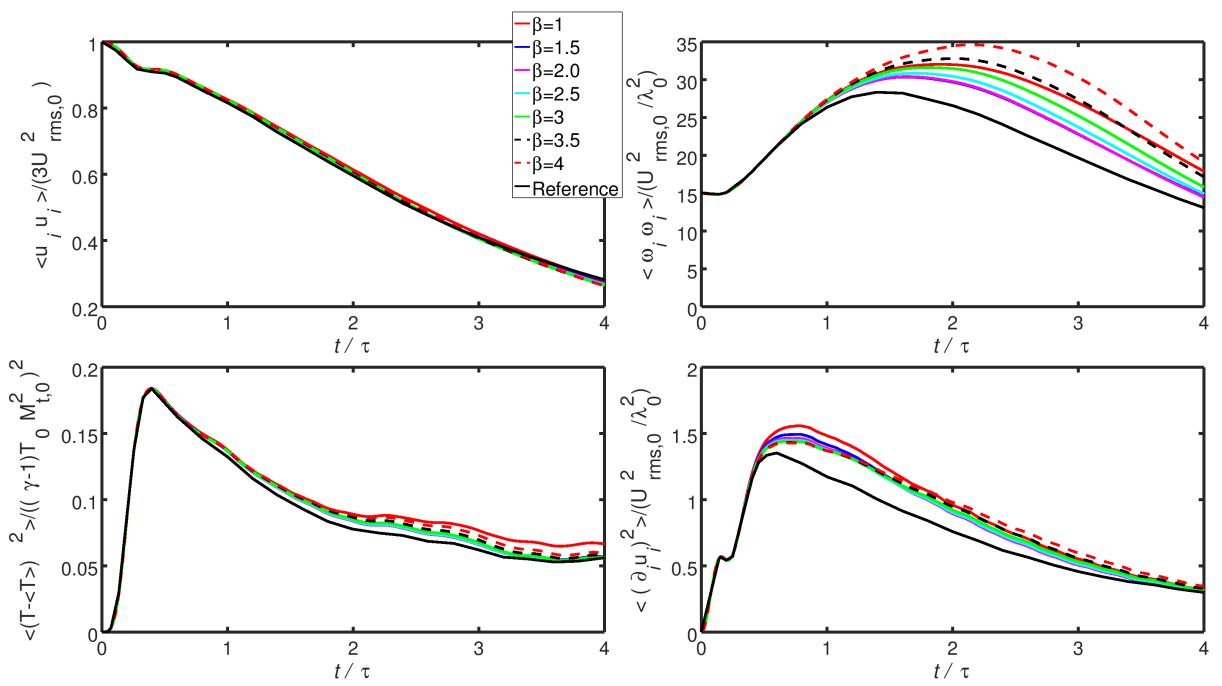

Fig. 6 3D Isotropic turbulence with $64^{3}$ grid points. Eight order accurate entropy split method. Evolution of kinetic energy (upper left), enstrophy (upper right), temperature variance (lower left), and dilatation (lower right) for different values of parameter $\beta$. Reference computed on $256^{3}$ grid points and filtered down to $64^{3}$ resolution.

Figure 6 shows the time evolution of the domain-averaged kinetic energy (upper left), enstrophy (upper right), temperature variance (lower left), and dilatation (lower right). Results with different values of the entropy splitting parameter $\beta$ are shown in different colors. The computations were done on 
a grid with a coarse DNS grid of $64^{3}$ points, using C08ES as spatial discretization and RK4 in time. The coarse grid DNS solutions are compared with the reference DNS solution using a $256^{3}$ grid filtered down to the $64^{3}$ grid. The figure shows that there is no single value of $\beta$ that is optimal for all four quantities plotted, but that $\beta \approx 2$ seems to be a good choice for overall accuracy.

The computed quantities in Fig. 6 that involve derivatives (enstrophy and dilitation) are visibly larger than the reference solution. In order to suppress unphysical oscillations, which are the likely reason for this behavior, Fig. 7 shows the same computations as in Fig. 6, but with a non-linear filter postprocessing the solution after each time step. The WENO7 filter using a wavelet sensor with $\kappa=0.4$, developed in [20], was used.
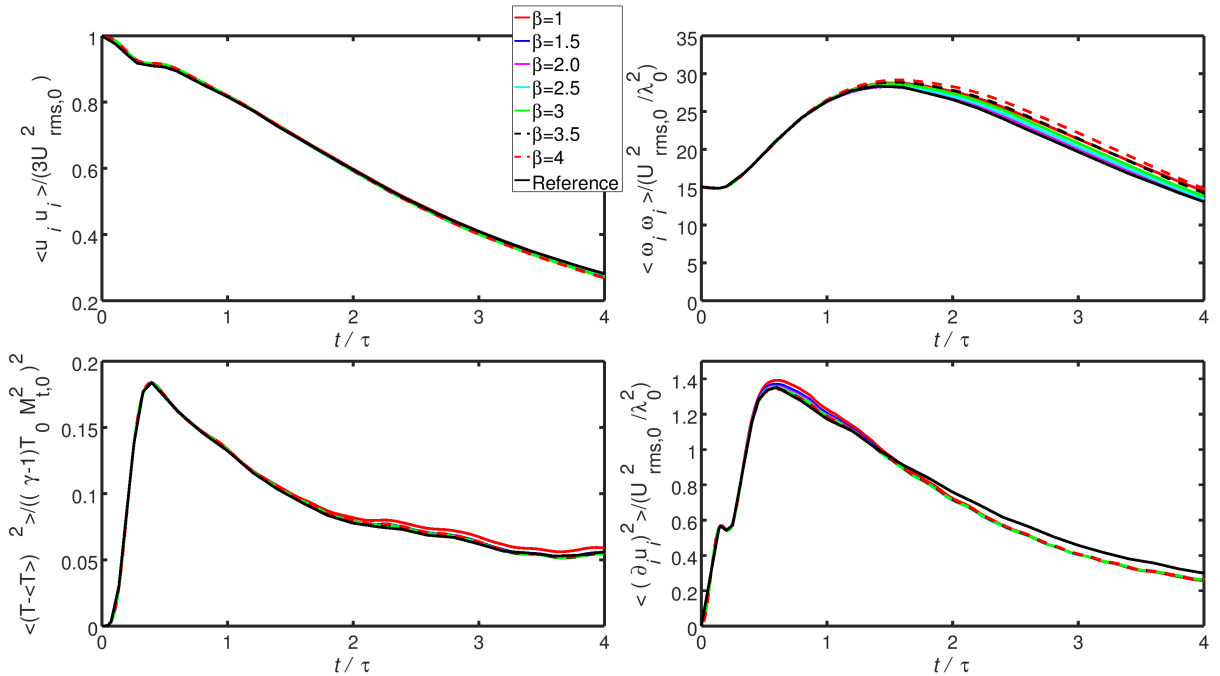

Fig. 7 3D Isotropic turbulence with $64^{3}$ grid points: Same computation as in Fig. 6 , but with WENO7FI postprocessing the solution after each time step.

Figure 7 shows that the filtered solution conforms better to the reference solution, and that values around $\beta=2$ perform well. All are in the presence of the WENO7 filter.

The computations in Fig. 6 are repeated, but with order of accuracy ten in Fig. 9. Similarly, Fig. 10 shows the tenth-order solution nonlinearly filtered after each time step with the dissipative part of the WENO9 scheme. The computation used the same flow sensor as used for the computation in Fig. 7.

Finally, Fig. 8 gives a comparison between the tenth-order entropy split scheme with $\beta=2$ (red color) and the tenth-order entropy conservative 

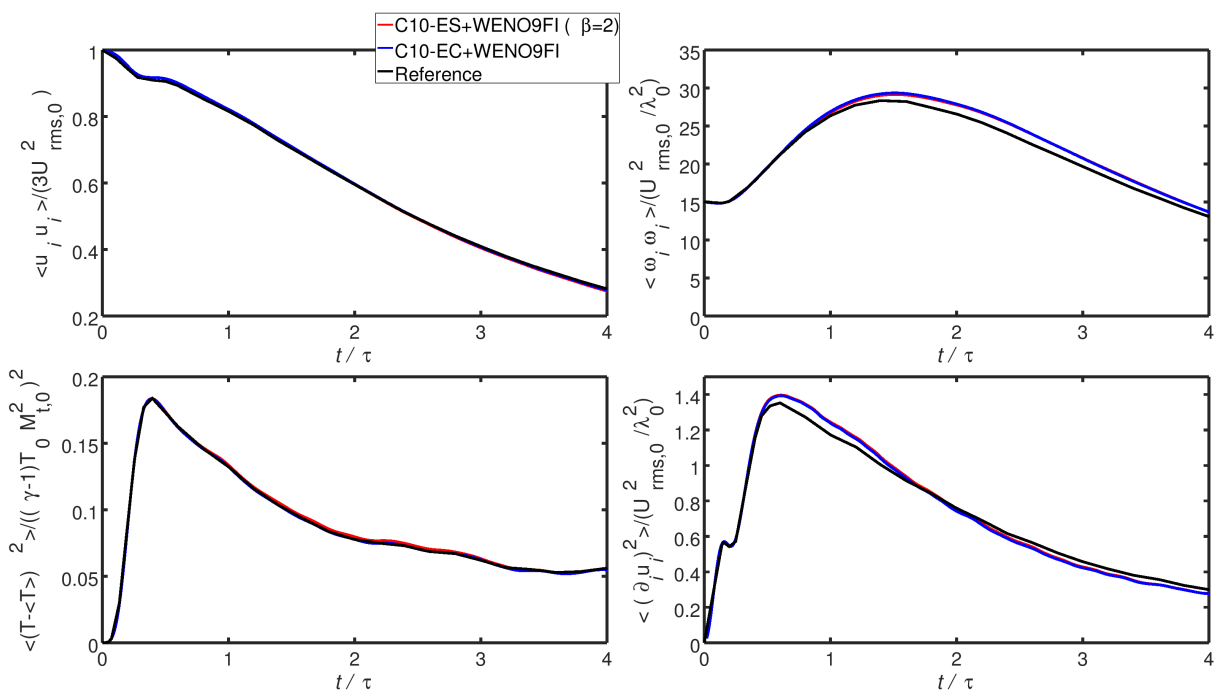

Fig. 8 3D Isotropic turbulence with $64^{3}$ grid points: Comparison C10ES+WENO9FI (red) and C10-EC+WENO9FI (blue).
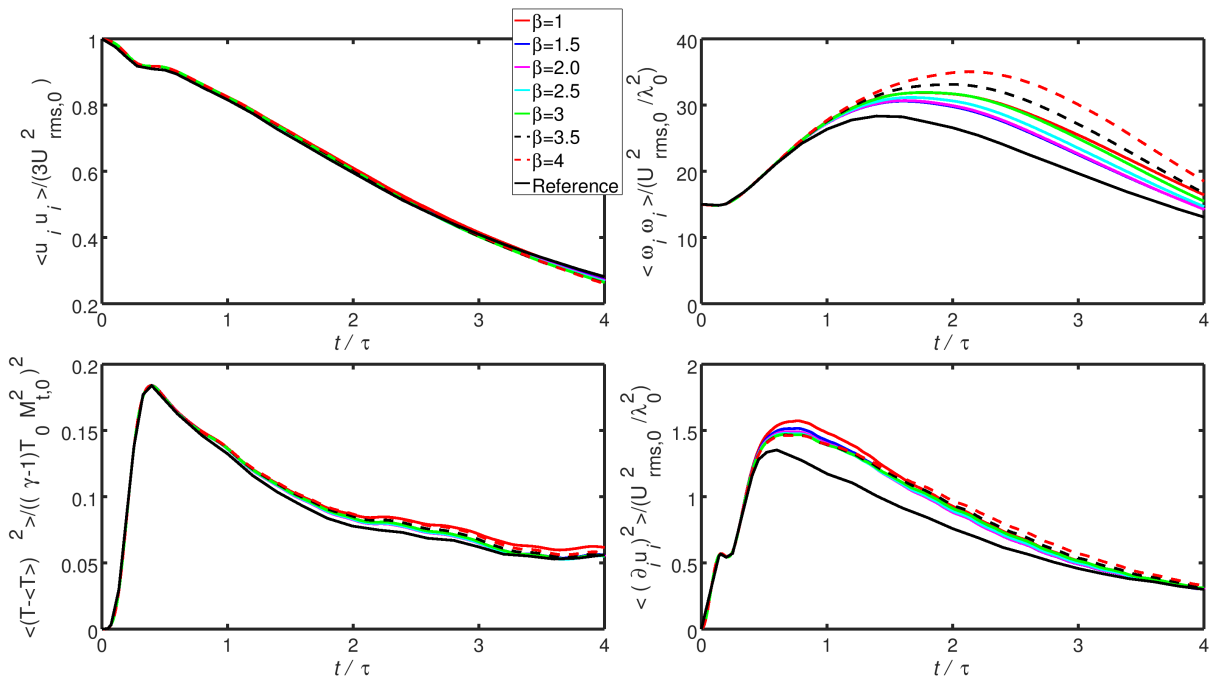

Fig. 9 3D Isotropic turbulence with $64^{3}$ grid points: Same computation as in Fig. 6, but with tenth-order of accuracy. 

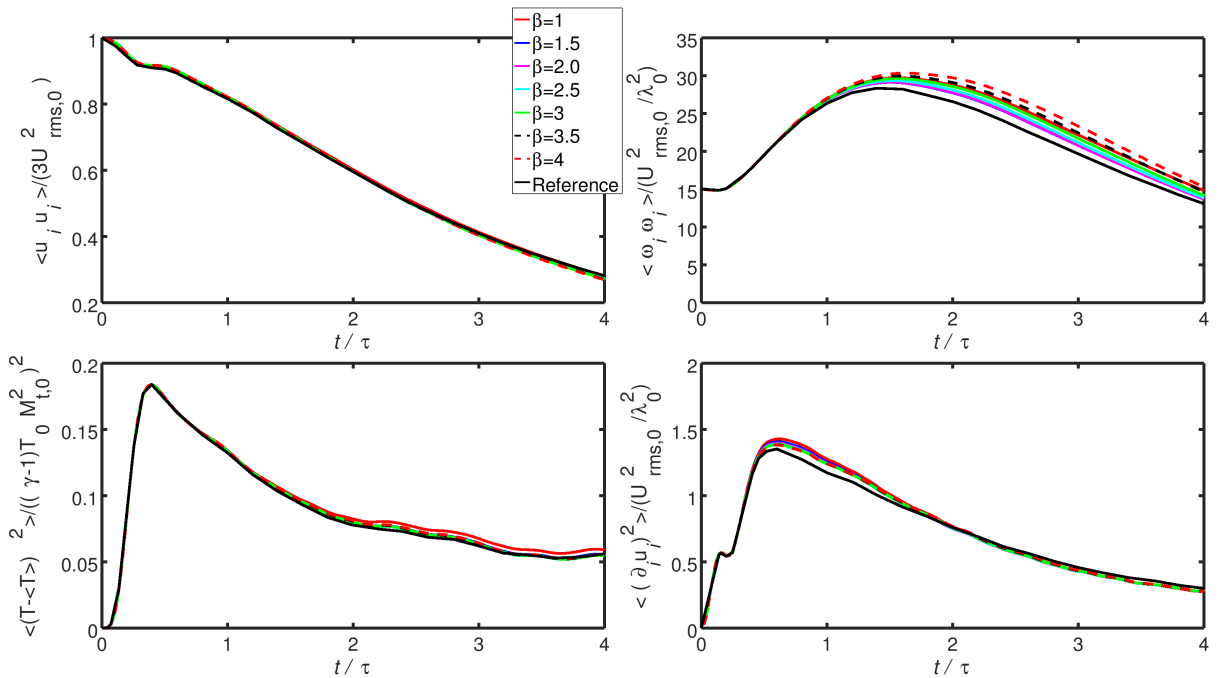

Fig. 10 3D Isotropic turbulence problem with $64^{3}$ grid points: Same computation as in Fig. 9, but with WENO9FI postprocessing the solution after each time step.

scheme (blue color). The entropy conservative scheme is in conservation form and uses the entropy $-\frac{\rho}{\gamma-1} \ln \left(p \rho^{-\gamma}\right)$; see [25] for a detailed description. The two solutions (entropy split and entropy conservative) in Fig. 8 are very close; it is hard to distinguish them in the figure.

The computations in Fig. 6 are repeated, but with order of accuracy ten in Fig. 9. Similarly, Fig. 10 shows the tenth-order solution nonlinearly filtered after each time step with the dissipative part of the WENO9 scheme. The computation used the same flow sensor as used for the computation in Fig. 7.

Figure 11 shows the comparison of two splitting methods (DS and KGS), ES (entropy splitting \& entropy stable) and EC (entropy conservative) using the same nonlinear filter. The time evolutions of the domain averaged kinetic energy (upper left), enstrophy (upper right), temperature variance (lower left), and dilatation (lower right) are compared. All four forms of the nonlinear filter method provide similar resolution. All four schemes without the nonlinear filter are stable but not as accurate as the nonlinear filter versions.

Over all, DS splitting is slightly less CPU intensive than ES. KGS skewsymmetric splitting is more CPU intensive than DS and ES. The EC method is around two times more expensive than DS. In addition, as the order of these methods increases, the gain in efficiency (CPU) of entropy split schemes increases. 

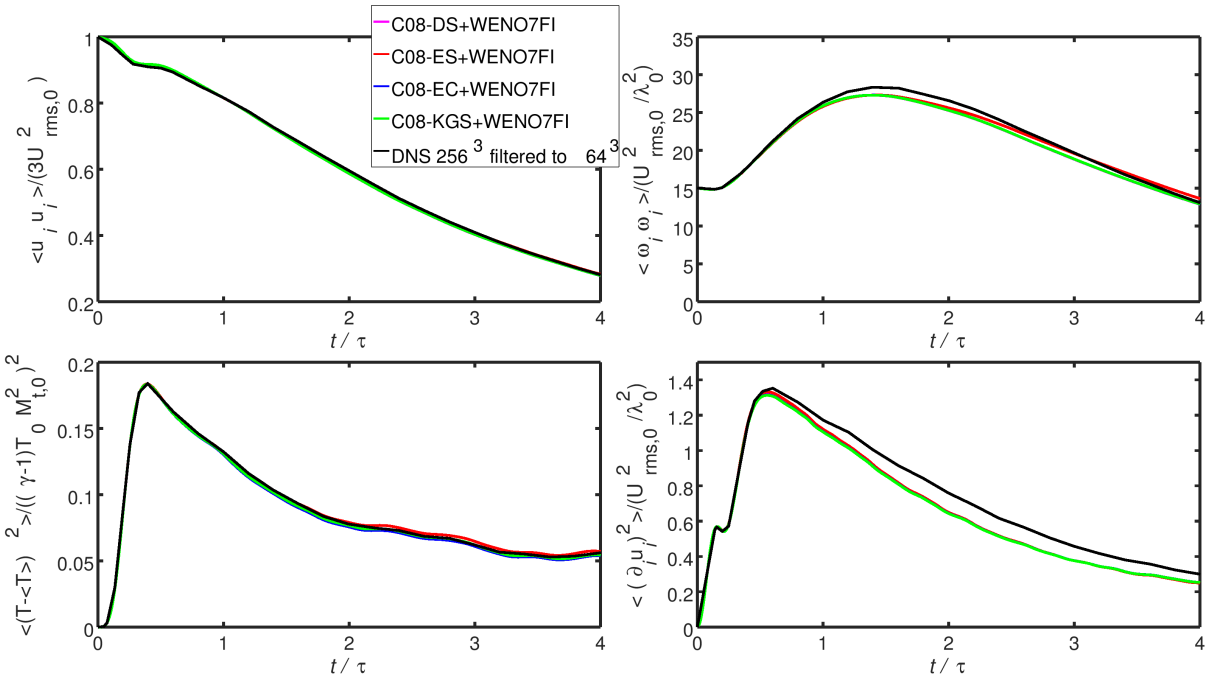

Fig. $113 \mathrm{D}$ Isotropic turbulence problem with $64^{3}$ grid points. Comparison of two splitting methods (DS and KGS), ES (entropy splitting \& entropy stable) and EC (entropy conservative) using the same nonlinear filter. Evolution of kinetic energy (upper left), enstrophy (upper right), temperature variance (lower left), and dilatation (lower right). DNS computed on $256^{3}$ grid points and filtered down to $64^{3}$ resolution is considered as the reference solution.

\section{Conclusions}

This paper revisits entropy split schemes for compressible gas dynamics and shows (in the current terminology) that entropy split schemes are entropy stable for high order spatial central schemes. Although entropy split methods are not in conservation form, Section 9 showed that they perform well on problems with shocklets with no indication of wrong shock speed. Over all, the Tadmor-type entropy conservative method of comparable order is approximately two times more CPU intensive than the entropy split scheme. In addition, as the order of these methods increases, the gain in efficiency (CPU) by entropy split schemes increases.

The entropy split scheme has been generalized from perfect gas to thermally perfect gas and gas flows consist of a linear combination of perfect gases $[34,37]$. These high order schemes have been formulated in time varying deforming curvilinear grids with free-stream preservation [34, 21]. It is noted that the Hughes et al. formulation [9] using the Harten's idea [8] but solving the flow equations in nonconservative form in terms of the entropy variables is completely different from the entropy split schemes. The entropy split schemes solve the entropy splitting form of the Euler flux derivatives 
consisting of a conservative and a non-conservative portion in terms of the entropy variables.

A natural extension of the entropy split scheme would be to define it for the logarithmic entropy $E=-\rho \log \left(p \rho^{-\gamma}\right)$. This cannot be done by the technique described here since the logarithmic entropy does not satisfy the homogeneity property (11). It is noted that it would be interesting, but is not possible by the straightforward type of entropy splitting described here for the ideal MHD. Although the entropy (9) is a valid entropy for MHD, the corresponding entropy variables do not satisfy the homogeneity (11) property. A completely different type of entropy split method that does not rely on homogeneity would be needed to treat these two cases.

The use of the Harten entropies (9) to construct Tadmor-type of entropy conserving schemes in conservative form is also shown for both gas dynamics and MHD by using the same technique as used for the logarithmic entropy in $[35,25]$. The CPU for the resulting entropy conserving scheme would be similar to the logarithmic entropy in [25]. As can be seen, the derivation using the entropies (9) to construct entropy conserving schemes follows naturally the steps in [25]. It is about twice more CPU intensive than the entropy split scheme for the same central spatial discretization.

Acknowledgements Financial support from the NASA TTT/RCA program for the second author is gratefully acknowledged. The authors are grateful to Dr. Alan Wray of NASA Ames Research Center for the numerous invaluable discussions throughout the course of this work.

\section{References}

1. Bonito, A., Guermond, J-L., and Popov, B.: Stability Analysis of Explicit Entropy Viscosity Methods for Nonlinear Scalar Conservation Equations, Math Comput 83 (287), DOI: 10.1090/S0025-5718-2013-02771-8, May 2014.

2. Bohm, M., Winters, A.R., Gassner, G.J., Derigs, D., Hindenlang, F., Saur, J.:An Entropy Stable Nodal Discontinuous Galerkin Method for the Resistive MHD Equations. PartI Theoory and Numerical Verification. Xiv:1802.07341v2 [math.NA] May 2018.

3. Ducros, F., Laporte, F., Soulères, T., Guinot, V., Moinat, P., Caruelle, B.: Highorder fluxes for conservative skew-symmetric-like schemes in structured meshes: application to compressible flows, J. Comput. Phys. 161, 114-139 (2000).

4. Fisher, T.C., Carpenter, M.H.: High-order entropy stable finite difference schemes for nonlinear conservation laws: Finite Domains. J. Comput. Phys. 252, 518-557 (2013).

5. Fjordholm, U.S., Mishra, S., Tadmor, E.: Arbitrary High-order Accurate Entropy Stable Essentially Nonoscillatory Schemes for Systems of Conservation Laws. Siam J. Numer. Anal. 50, 544-573 (2012).

6. Friedrich, L., Winters, A., DelRey Fernandez, D., Gassner, G., Paarsani, M., Carpenter, M.: An Entropy Stable h/p Non-conforming Discountinuous Galerkin Method with Summation-by-Parts Property. J Sci Comput 77, 689-725, (2018). 
7. Gerritsen, M, Olsson, P.: Designing an efficient solution strategy for fluid flows. I. A stable high order finite difference scheme and sharp shock resolution for the Euler equations. J. Comput. Phys. 129, 245-262 (1996).

8. Harten, A: On the symmetric form of systems for conservation laws with entropy: J. Comput Phys. 49, 151 (1983).

9. Hughes, T., Franca, L., and Mallet, M.: A new finite element formulation for computational fluid dynamics: K. Symmetric forms of the compressible Euler and Navier-Stokes equations and the second law of thermodynamics, Computer Methods in Applied Mechanics and Engineering, 54, 223-234 (1986).

10. Ismail, F., Roe, P.L.: Affordable, entropy-consistent Euler flux functions II: Entropy production at shocks. J. Comput Phys. 228, 5410-5436 (2009).

11. Johansson, S.: High Order Summation by Parts Operator Based on a DRP Scheme Applied to 2D, Technical Report 2004-050, Uppsala University, Sweden.

12. Kennedy, C.A. and Gruber, A.: Reduced Aliasing Formulations of the convective Terms Within the Navier-Stokes Equations. J. Comput. Phys., 227 1676-1700 (2008).

13. Kotov, D.V., Yee, H.C., Wray, A.A., Sjögreen, B., Kritsuk, A.G.: Numerical disipation control in high order shock-capturing schemes for LES of low speed flows. J. Comput. Phys. 307, 189-202 (2016).

14. LeFloch, P.G., Mercier, J.M., Rohde, C.,: Fully Discrete, Entropy Conservative Schemes of Arbitrary Order. SIAM J. Numer. Anal. 40, 1968-1992, (2002).

15. Olsson, P., Oliger, J.: Energy and maximum norm estimates for nonlinear conservation laws. RIACS Technical Report 94.01, (1994).

16. Parsani, M., Carpenter, M.H., Nielsen, E.J.: Entropy stable wall boundary conditions for the three-dimensional compressible Navier-Stokes equations. J. Comput. Phys. 292, 88-113 (2015).

17. Roanocha, H.: Comparison of some Entropy Conservative Numerical Fluxes for the Euler Equations. . Xiv:1701.02264v2 [math.NA], Nov. 2017.

18. Roanocha, H.: Generalized summation-by-Parts Operators and Variable Coefficients. Xiv:1705.10541v2 [math.NA], Feb. 2018.

19. Sandham, N.D., Li, Q., Yee, H.C.: Entropy splitting for high-order numerical simulation of compressible turbulence. J. Comput. Phys. 23, 307-322 (2002).

20. Sjögreen, B., Yee, H.C.: Multiresolution wavelet based adaptive numerical dissipation control for high order methods. J. Scientific Computing, 20, 211-255 (2004).

21. Sjögreen, B., Yee, H.C., Vinokur, M.: On High Order Finite-Difference Metric Discretizations Satisfying GCL on Moving and Deforming Grids, J. comput. Phys., 265 211-220 (2014).

22. Sjögreen, B., Yee, H.C.: On skew-symmetric splitting and entropy conservation schemes for the Euler equations. In: Proceedings of ENUMATH09, June 29- July 2, Uppsala University, Sweden (2009).

23. Sjögreen, B., Yee, H.C.: Accuracy Consideration by DRP Schemes for DNS and LES of Compressible Flow Computations. Special issue in Computers \& Fluids in honor of Prof. Toro's 70th birthday, 159 (2017) 123-136.

24. Sjögreen, B., Yee, H.C.: Skew-Symmetric Splitting for Multiscale Gas Dynamics and MHD Turbulence Flows. Extended version of Proceedings of ASTRONUM2016, June 6-10, 2016, Monterey, CA, USA, submitted to J. Scientific Computing, 2018.

25. Sjögreen, B., Yee, H.C.:High order entropy conservative central schemes for wide ranges of compressible gas dynamics and MHD flows. J. Comput. Phys., 364, 153-185 (2018).

26. Sjögreen, B., Yee, H.C.: Two Decades Old Entropy Stable Methods for the Euler Equations Revisited. Proceedings of the ICOSAHOM-2018, July 9-13, 2018, London, UK. 
27. Sandham, N.D., Li, Q., Yee, H.C.: Entropy Splitting for High-Order Numerical Simulation of Compressible Turbulence, 178, 307-322 (2002).

28. Svärd, M., Mishra, S.: Shock Capturing Artificial Dissipation for High-Order Finite Difference Schemes. J. Sci. Comput., 39 (2009) 454-484.

29. Tadmor, E.: Numerical Viscosity and the Entropy Condition for Conservative Difference Schemes. Math. Comput., 43, 369-381 (1984).

30. Tadmor, E.: The Numerical viscosity of entropy stable schemes for systems of conservation laws. I. Math. of Comput., 49, 91-103 (1987).

31. Tadmor, E.: Entropy Stability Theory for Difference Approximations of Nonlinear Conservation Laws and Related Time-Dependent Problems. Acta Numerica 12, 451-512 (2003).

32. Taylor, G., Green, A.: Mechanism of the Production of Small Eddies from Large Ones. Proc. R. Soc. Lond. A 158, 499-521 (1937).

33. Tauber, E., Sandham, N.D.,: Comparison of three large-eddy simulatitons of shock-induced turbulent separation bubbles: Shock waves, 19, 469-478 (2009).

34. Vinokur, M., Yee, H.C.: Extension of Efficient Low Dissipation High-Order Schemes for 3D Curvilinear Moving Grids. Front. Comput. Fluid Dyn. 12964 (2002); Also, Proceedings of the Robert MacCormack 60th Birthday Conference, June268, 2000, Half Moon Bay, CA, NASA/TM-2000-209598.

35. Winters, A.R., Gassner, G.J.: Affordable, entropy conserving and entropy stable flux functions for the ideal MHD equations. J. Comput. Phys. 304, 72-108 (2016).

36. Yee, H. C., Sandham, N.D., Djomehri, M. J.: Low-Dissipative High Order ShockCapturing Methods Using Characteristtic-Based Filters. J. Comput. Phys., 150, 199-238 (1999).

37. Yee, H. C., Vinokur, M, Djomehri, M. J.: Entropy Splitting and Numerical Dissipation. J. Comp. Phys. 162, 33-81 (2000).

38. Yee, H. C., Sjögreen, B.: Development of low dissipative high order filter schemes for multiscale Navier-Stokes and MHD systems. J. Comput. Phys., 225 910-934 (2007).

39. Yee, H. C., Sjögreen, B.: High Order Filter Methods for Wide Range of Compressible flow Speeds. Proceedings of the ICOSAHOM09, June 22-26, 2009, Trondheim, Norway. 